\title{
Complex Picture Fuzzy N-Soft Sets and Their Decision Making Algorithm
}

\author{
Tahir Mahmood \\ International Islamic University \\ Ubaid ur Rehman ( $\square$ ubaid5@outlook.com ) \\ International Islamic University https://orcid.org/0000-0002-5361-6592 \\ Jabbar Ahmmad \\ International Islamic University
}

\section{Research Article}

Keywords: Complex picture fuzzy N-soft sets, complex picture fuzzy sets, $\mathrm{N}$-soft sets, soft sets, picture fuzzy soft sets, decision making.

Posted Date: May 3rd, 2021

DOl: https://doi.org/10.21203/rs.3.rs-278288/v1

License: (c) (i) This work is licensed under a Creative Commons Attribution 4.0 International License. Read Full License 


\title{
Complex Picture Fuzzy N-Soft Sets and Their Decision Making Algorithm
}

\author{
${ }^{1}$ Tahir Mahmood, ${ }^{1}$ Ubaid ur Rehman, and Jabbar Ahmmad ${ }^{1}$ \\ ${ }^{1}$ Department of Mathematics and Statistics, International Islamic University Islamabad, Pakistan. \\ tahirbakhat@iiu.edu.pk; ubaid5@outlook.com; jabbarahmad1992@gmail.com
}

Correspondence: ubaid5@outlook.com.

\begin{abstract}
We establish in this manuscript a new model complex picture fuzzy N-soft sets (CPFN-SSs) which is the fusion of complex picture fuzzy sets (CPFSs) along with N-soft sets (N-SSs) and a novel definition of CPFSs which is a mixture of complex fuzzy sets (CFSs) and picture fuzzy sets (PFSs). In CPFN-SS notions of N-SSs, fuzzy N-SSs (FN-SSs), intuitionistic FN-SSs (IFN-SSs), picture FN-SSs (PFN-SSs) complex FN-SSs (CFN-SS), complex IFN-SSs (CIFN-SSs) are generalized. We also talk about few basic operations on CPFNSSs with the assistance of examples. Additionally, we establish two kinds of union (extended and restricted) and intersection (extended and restricted) of CPFN-SSs and illustrate them through examples. We also establish a complement of CPFN-SSs along with an example. Furthermore, we define an algorithm to solve the data in the environment of CPFN-SSs, and then we represent two real-world applications (performance assessment of e-waste recycling program and predication about the champions of FIFA world cup 2022 through audience poll) of our established model in decision-making (DM) to show the advantages and usefulness of the CPFN-SSs. In the end, we represent a comparison of our established model with some existing methods which shows that our established model is more general than existing ones.
\end{abstract}

Keywords: Complex picture fuzzy N-soft sets, complex picture fuzzy sets, N-soft sets, soft sets, picture fuzzy soft sets, decision making.

\section{Introduction}

In the real-life, there are several difficult issues in management science, engineering, environment, economics, and social science. They are described with dubiousness, fuzziness, and imprecision. We can't effectively use the old methods to manage these issues in the light of the fact that there are different kinds of uncertainties associated with these issues. However, there are several notions like notion of probability, the notion of FSs [1], the notion of interval mathematics [2], the notion of rough sets [3-7] to be supposed as mathematical instruments to manage uncertainties. In these notions, FSs got a lot of attraction from the researchers and it has been generalized. This notion is valuable apparatus in DM [8]. In FSs notion the positive grade belongs to $[0,1]$. Regardless, because of the uncertainty degree generally, it is not true that the negative grade in FSs is equivalent to 1 minus the positive grade. To clarify this Atanassov [9] established the notion of intuitionistic FSs (IFSs), which is a reasonable generalization of FSs. Subsequently, Cuong [10] introduced the notion of picture FSs (PFSs) which is made by the positive grade, neutral grade or abstain grade, negative grade, and refusal grade, accordingly PFSs can describe the views of decision makers more precisely than the FS and IFS. Abdullah et al. [11] defined a novel approach based on sine trigonometric picture fuzzy aggregation operator and their application in decision support system.

Another innovative idea complex FSs (CFSs) is a modification of FSs established by Ramot et al. [12], where the positive grade $\rho$ instead of being real-valued grade belong to closed interval $[0,1]$ is replaced by 
complex-valued grade $\phi$. The vital aspect of CFSs is the existence of phase term and its grade. This provides those CFSs wavelike properties which might result in constructive and destructive intervention relying upon the phase value. Numerous examples are presented in [13], which describe the usage of such CFSs. Tamir et al. [14] defined a new interpretation of CFSs. Imtiaz et al. [15] introduced the structural properties of CFSs and their applications. Akram et al. [16] initiated the idea of complex PFS (CPFS) by adding the neutral grade and negative grade to the definition of CFS creates CPFS a modification of CFS and complex IFS (CIFS). Mahmood et al. [17] presented the notion of complex hesitant FSs (CHFSs). The notion of complex dual hesitant FSs (CDHFSs) was established by Mahmood et al. [18].

All the above notions have their drawbacks and restriction to conquer these challenges Molodtsov [19] first and foremost established a novel mathematical instrument named SS notion to manage vagueness and uncertainty. This notion is valuable apparatus in numerous applications like game theory, DM, and measurement theory. Maji et al. [20] developed some algebraic operations in SS. Ali et al. [21] presented few revised operations in SS. Anyhow, the data often comes across in a complicated shape. A great number of authors have grown new models to beat such sort of information and combine the benefits of the established models. These models are created to determine a huge range of DM problems as they have a greater number of benefits than the original ones. The SS model can be mixed with various mathematical models. Maji et al. [22] described the notion of fuzzy SS (FSS) by maxing the notion of FS and SS. Feng et al. [23] presented the FSS approach to the ideal theory of regular AG-groupoids. Additionally, Maji et al. $[24,25]$ defined the idea of intuitionistic FSS (IFSS) which is the fusion of IFS [9] and SS. By fusing the interval-valued FS (IVFS) [2, 26] and SS, Yang et al. [27] initiated the idea of the interval-valued FSS (IVFSS). Khan et al. [28] presented generalized PFSSs and initiated some improved operations for PFSSs. Khan et al. [29] presented the generalized mutli-fuzzy bipolar SSs and its application in DM.

$\mathrm{N}$-SSs is the idea of a parameterized representation of objects of a universe that relies upon a finite number of ordered grades. Fatimah et al. [30] were inspired by real issues. The vast majority of the researchers in SS motivated model performed on the initial binary setting (assessments are either 0 or 1 ) or otherwise, the situation where assessments are real numbers between 0 and 1 [31]. But we frequently get the information with a non-binary but discrete structure in everyday life. E.g. in the representation of ranking or rating positions or in social judgment system [32]. From everyday life we can see many examples of rating system where ranking take shape in the form of the number of stars or dots etc. which can demonstrate in the shape of natural numbers. More, Herawan and Deris [33] showed $n$ binary-valued data system in SSs, where every attribute has its particular ranking, when contrasted with the rating order presented by Chen et al. [34]. Rather than ratings as evaluations Ali et al. [35] formed a rating system between the elements of SSs parameters. Akram et al. [36] interpreted the notion of FN-SS. The idea of IFN-SS is described by Akram et al. [37].

There are various generalizations of N-SS to deal with uncertainty and vagueness such as FN-SS, IFN-SS, etc. But when a decision-maker gives the data of the type like yes, no, abstain, or neutral along with twodimensional information in the grading system then these existing notions are not capable to cope with it. To resolve such type of complication we define the notion of CPFN-SS which is a perfect tool to cope with such type of issues. The voting system or audience poll through voting with two-dimensional information is the best real-life example of such issues, where we divide the voters into 4 parts (vote for, abstain or neutral, vote against, and refusal) and we want two-dimensional data of every electoral candidate then the 
existing notions are not able to give us any result but CPFN-SS can solve such type of DM. CPFN-SS generalize N-SSs, FN-SSs, IFN-SSs, PFN-SSs, CFN-SS, and CIFN-SSs as follows

1. If we neglect the complex neutral grade $(\mathrm{CnG})$ then CPFN-SS will transform to IFN-SS.

2. If we neglect the $\mathrm{CnG}$, and complex negative grade (CNG) then CPFN-SS will transform to CFNSS.

3. If we take the phase term zero in the complex positive grade (CPG), $\mathrm{CnG}$, and CNG then the CPFNSS will transform to PFN-SS.

4. If we neglect the CnG and take the phase term zero in the CPG and CNG then the CPFN-SS will transform to IFN-SS.

5. If we neglect the CnG and CNG and take the phase term zero in the CPG then the CPFN-SS will transform to FN-SS.

6. If we neglect the CPG, CnG, and CNG then the CPFN-SS will transform to N-SS.

The rest of the article is organized as: In section 2 for better description and understanding we review some fundamental definitions like FSs, IFS, PFSs, CPFSs, SSs, FSSs, IFSSs, PFSSs N-SSs, and IFN-SS along with some properties. In section 3, we establish the novel notion of CPFS and define its operations. In section 4, we initiated the notion of our model CPFN-SS which is the fusion of CPFS and N-SS. In the same section, we establish some operations of CPFN-SS and explain them by examples. Further, in section 5, We initiate an algorithm to deal with the data in the environment of CPFN-SS and then provide two real-life examples to show the practicability and effectiveness of our established work. The comparison of CPFN-SS with some FN-SS and IFN-SS is presented in section 6. In the end, the conclusion of this article is presented in section 7 .

\section{Preliminaries}

For better understanding, in this section, we recall some underlying definitions like FSs, IFSs, PFSs, CPFSs, SSs, FSSs, IFSSs, PFSSs, N-SSs, and IFN-SS. We review the important properties of a few of the above underlying definitions. $Z \neq \emptyset$ will designate the set of a universe and $\beta$ will designate a set of attributes in this manuscript.

Definition 1: [1] A FS $\mathcal{H}$ is elaborated as

$$
\mathcal{H}=\left\{\left(z, \rho_{\mathcal{H}}(z)\right): z \in Z\right\},
$$

where $\rho_{\mathcal{H}}: \mathcal{Z} \rightarrow[0,1]$ is a positive grade. The pair $\mathcal{H}=\left(z, \rho_{\mathcal{H}}(\mathfrak{z})\right)$ is expresses the fuzzy number (FN)

Definition 2: [1] Suppose $\mathcal{H}=\left(z, \rho_{\mathcal{H}}(\mathfrak{z})\right)$ and $\mathcal{J}=\left(z, \rho_{\mathcal{J}}(\mathfrak{z})\right)$ are two FNs, then

1. $\mathcal{H}=\mathcal{J} \Leftrightarrow \rho_{\mathcal{H}}(\mathfrak{\jmath})=\rho_{\mathcal{J}}(\mathfrak{3}) ;$

2. $\mathcal{H} \subseteq \mathcal{J} \Leftrightarrow \rho_{\mathcal{H}}(3) \leq \rho_{\mathcal{J}}(3)$;

3. $\mathcal{H}^{c}=\left(3,1-\rho_{\mathcal{H}}(3)\right)$;

4. $\mathcal{H} \cup \mathcal{J}=\left(\mathfrak{\jmath}, \max \left(\rho_{\mathcal{H}}(\mathfrak{\jmath}), \rho_{\mathcal{J}}(\mathfrak{\jmath})\right)\right)$;

5. $\mathcal{H} \cap \mathcal{J}=\left(z, \min \left(\rho_{\mathcal{H}}(\mathfrak{\jmath}), \rho_{\mathcal{J}}(\mathfrak{\jmath})\right)\right)$.

Definition 3: [9] An IFS elaborated as

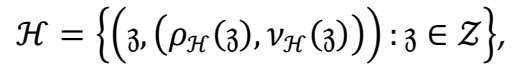

where $\rho_{\mathcal{H}}: Z \rightarrow[0,1]$, is a positive grade and $v_{\mathcal{H}}: Z \rightarrow[0,1]$ is negative grade with a condition that $0 \leq$

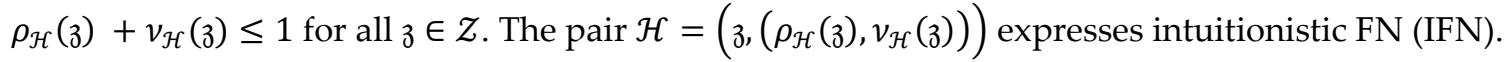




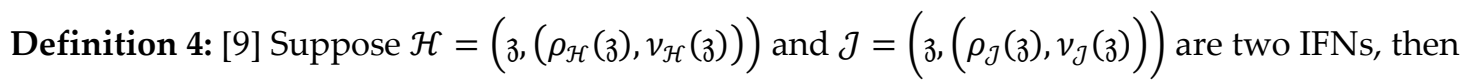

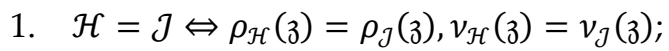

2. $\mathcal{H} \subseteq \mathcal{J} \Leftrightarrow \rho_{\mathcal{H}}(\mathfrak{\jmath}) \leq \rho_{\mathcal{J}}(\mathfrak{\jmath}), v_{\mathcal{H}}(\mathfrak{\jmath}) \geq v_{\mathfrak{J}}(\mathfrak{\jmath})$;

3. $\mathcal{H}^{c}=\left(\jmath, v_{\mathcal{H}}(\precsim), \rho_{\mathcal{H}}(\precsim)\right)$;

4. $\mathcal{H} \cup \mathcal{J}=\left(z, \max \left(\rho_{\mathcal{H}}(\mathfrak{z}), \rho_{\mathcal{J}}(\mathfrak{z})\right), \min \left(v_{\mathcal{H}}(\mathfrak{z}), v_{\mathcal{J}}(\mathfrak{z})\right)\right)$;

5. $\mathcal{H} \cap \mathcal{J}=\left(\jmath, \min \left(\rho_{\mathcal{H}}(\mathfrak{\jmath}), \rho_{\mathfrak{J}}(\mathfrak{\jmath})\right), \max \left(v_{\mathcal{H}}(\mathfrak{z}), v_{\mathcal{J}}(\mathfrak{\jmath})\right)\right)$.

Definition 5: [10] A PFS elaborated as

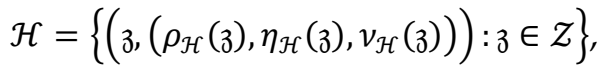

where $\rho_{\mathcal{H}}: Z \rightarrow[0,1]$, is a positive grade, $\eta_{\mathcal{H}}: Z \rightarrow[0,1]$ is a neutral grade and $v_{\mathcal{H}}: Z \rightarrow[0,1]$ is a negative grade along with a condition that $0 \leq \rho_{\mathcal{H}}(\xi)+\eta_{\mathcal{H}}(\xi)+v_{\mathcal{H}}(z) \leq 1$ for all $z \in Z$. The triplet $\mathcal{H}=$ $\left(\jmath,\left(\rho_{\mathcal{H}}(3), \eta_{\mathcal{H}}(3), v_{\mathcal{H}}(3)\right)\right)$ expresses a picture FN $(\mathrm{PFN})$.

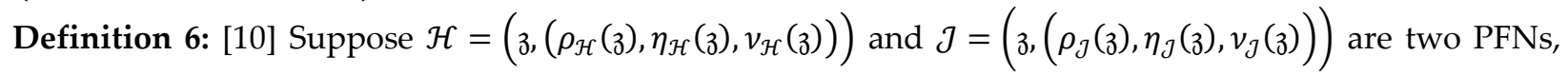
then

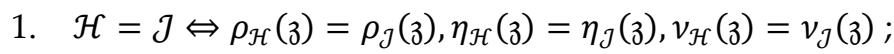

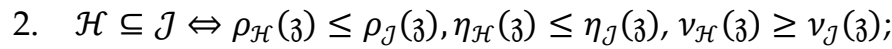

3. $\mathcal{H}^{c}=\left(3, v_{\mathcal{H}}(3), \eta_{\mathcal{H}}(3), \rho_{\mathcal{H}}(3)\right)$;

4. $\mathcal{H} \cup \mathcal{J}=\left(z, \max \left(\rho_{\mathcal{H}}(\mathfrak{z}), \rho_{\mathcal{J}}(\mathfrak{z})\right), \min \left(\eta_{\mathcal{H}}(\mathfrak{z}), \eta_{\mathcal{J}}(\mathfrak{z})\right), \min \left(v_{\mathcal{H}}(\mathfrak{z}), v_{\mathfrak{J}}(\mathfrak{z})\right)\right) ;$

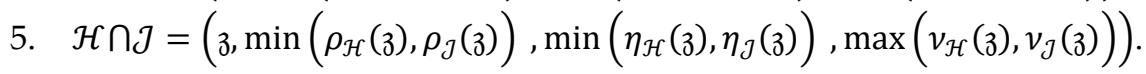

Definition 7: [19] A pair $(\Delta, \Re)$ is called a SS over $Z$ where $\Delta: \Re \rightarrow P(Z)$, be set valued function and $\Re \subseteq \Re$. Definition 8: [21] Suppose $\left(\Delta_{1}, \Re_{1}\right)$ and $\left(\Delta_{2}, \Re_{2}\right)$ are two SSs with $\Re_{1} \cap \Re_{2} \neq \varnothing$, then their restricted union and intersection are interpreted as:

$$
\begin{gathered}
\left(\Delta_{1}, \Re_{1}\right) \cup_{r}\left(\Delta_{2}, \Re_{2}\right)=\left(\mathfrak{f}, \Re_{1} \cap \Re_{2}\right) \forall \mathfrak{r} \in \Re_{1} \cap \Re_{2} \Rightarrow \mathfrak{f}(\mathfrak{r})=\Delta_{1}(\mathfrak{r}) \cup \Delta_{2}(\mathfrak{r}) . \\
\left(\Delta_{1}, \Re_{1}\right) \cap_{r}\left(\Delta_{2}, \Re_{2}\right)=\left(\mathfrak{g}, \mathfrak{R}_{1} \cap \mathfrak{R}_{2}\right) \text { where } g(\mathfrak{r})=\Delta_{1}(\mathfrak{r}) \cap \Delta_{2}(\mathfrak{r}) \forall \mathfrak{r} \in \Re_{1} \cap \mathfrak{R}_{2} .
\end{gathered}
$$

Definition 9: [21] Suppose $\left(\Delta_{1}, \Re_{1}\right)$ and $\left(\Delta_{2}, \Re_{2}\right)$ are two SSs with $\Re_{1} \cap \Re_{2} \neq \emptyset$, then their extended union and intersection are interpreted as:

$$
\begin{aligned}
& \left(\Delta_{1}, \Re_{1}\right) \cup_{e}\left(\Delta_{2}, \Re_{2}\right)=\left(f, \Re_{1} \cup \Re_{2}\right) \\
& \text { where } \forall \mathfrak{r} \in \mathfrak{R}_{1} \cup \Re_{2} \Rightarrow f(\mathfrak{r})=\left\{\begin{array}{cc}
\Delta_{1}(\mathfrak{r}) & \text { if } \mathfrak{r} \in \mathfrak{R}_{1} \backslash \mathfrak{R}_{2} \\
\Delta_{2}(\mathfrak{r}) & \text { if } \mathfrak{r} \in \mathfrak{R}_{2} \backslash \mathfrak{R}_{1} \\
\Delta_{1}(\mathfrak{r}) \cup \Delta_{2}(\mathfrak{r}) & \mathfrak{r} \in \mathfrak{R}_{1} \cup \mathfrak{R}_{2}
\end{array}\right. \\
& \left(\Delta_{1}, \Re_{1}\right) \cap_{e}\left(\Delta_{2}, \Re_{2}\right)=\left(g, \Re_{1} \cup \Re_{2}\right) \\
& \text { where } \forall \mathfrak{r} \in \Re_{1} \cup \Re_{2} \Rightarrow g(\mathfrak{r})=\left\{\begin{array}{cc}
\Delta_{1}(\mathfrak{r}) & \text { if } \mathfrak{r} \in \Re_{1} \backslash \Re_{2} \\
\Delta_{2}(\mathfrak{r}) & \text { if } \mathfrak{r} \in \Re_{2} \backslash \Re_{1} \\
\Delta_{1}(\mathfrak{r}) \cap \Delta_{2}(\mathfrak{r}) & \mathfrak{r} \in \Re_{1} \cup \Re_{2}
\end{array}\right.
\end{aligned}
$$

Definition 10: [22] A pair $\left(\Delta^{\prime}, \Re\right)$ characterizes a FSS over $Z$ if $\Delta^{\prime}: \Re \rightarrow \mathcal{F} \mathcal{S}(\mathcal{Z}), \Re \subseteq \Re$, where $\mathcal{F} \mathcal{S}(Z)$ signifies the set of all FS of $Z$.

Definition 11: [24] A pair $\left(\Delta^{\prime \prime}, \Re\right)$ characterizes an IFSS over $Z$ if $\Delta^{\prime \prime}: \Re \rightarrow I \mathcal{F} \mathcal{S}(Z), \Re \subseteq ß$, where $I \mathcal{F} \mathcal{S}(Z)$ signifies the set of all IFS of $Z$.

Definition 11: [28] Suppose $\mathcal{P F} \mathcal{S}(Z)$ is a set of all PFSs over $Z$, then a pair $\left(\Delta^{\prime \prime \prime}, \Re\right)$ is said to be PFSS over $Z$, where $\Delta^{\prime \prime \prime}: \Re \rightarrow \mathcal{P F} \mathcal{S}(\mathcal{Z}), \Re \subseteq ß$.

Definition 12: [30] Suppose a universal set $Z$ and the attributes set $\beta$, a triplet $(\Delta, \mathcal{P}, N)$ is called N-SS over $Z$, where $\Delta: \Re \rightarrow 2^{Z \times \mathfrak{X}}=P(Z \times \mathfrak{X}), \mathfrak{R} \subseteq ß$, with the property that for each $\mathfrak{r} \in \mathfrak{R}$ and $\exists$ a unique $\left(\left(\mathfrak{z}, \mathfrak{x}_{\mathfrak{r}}\right) \in P(\mathcal{Z} \times \mathfrak{X})\right)$ such that $\left(\mathfrak{z}, \mathfrak{x}_{\mathfrak{r}}\right) \in \Delta(\mathfrak{x}), \mathfrak{z} \in \mathcal{Z}, \mathfrak{x}_{\mathfrak{r}} \in \mathfrak{X}=\{0,1,2, \ldots, N-1\}$ be ordered grades set. 
Definition 13: [30] Suppose $\Gamma_{1}=\left(\Delta_{1}, \Re_{1}, N_{1}\right)$ and $\Gamma_{2}=\left(\Delta_{2}, \Re_{2}, N_{2}\right)$ are two N-SSs with $\Re_{1} \cap \Re_{2} \neq \emptyset$, then their restricted union and intersection are interpreted as:

$$
\Gamma_{1} \cup_{R} \Gamma_{2}=\left(\Delta_{1}, \Re_{1}, N_{1}\right) \cup_{R}\left(\Delta_{2}, \Re_{2}, N_{1}\right)=\left(h, \Re_{1} \cap \Re_{2}, \max \left(N_{1}, N_{2}\right)\right)
$$

where $\forall \mathfrak{r} \in \Re_{1} \cap \Re_{2} \& z \in Z \Rightarrow\left(\mathfrak{z}, x_{\mathfrak{r}}\right) \in h(\mathfrak{r}) \Leftrightarrow x_{\mathfrak{r}}=\max \left(x_{\mathfrak{r}}^{1}, x_{\mathfrak{r}}^{2}\right)$, if $\left(\mathfrak{z}, x_{\mathfrak{r}}^{1}\right) \in \Delta_{1}(\mathfrak{r}) \&\left(\mathfrak{z}, x_{\mathfrak{r}}^{2}\right) \in \Delta_{2}(\mathfrak{r})$.

$$
\Gamma_{1} \cap_{R} \Gamma_{2}=\left(\Delta_{1}, \Re_{1}, N_{1}\right) \cap_{R}\left(\Delta_{2}, \Re_{2}, N_{2}\right)=\left(\ell, \Re_{1} \cap \Re_{2}, \min \left(N_{1}, N_{2}\right)\right)
$$

where $\forall \mathfrak{r} \in \Re_{1} \cap \Re_{2} \& \mathfrak{z} \in Z \Rightarrow\left(\mathfrak{\jmath}, \mathfrak{x}_{\mathfrak{r}}\right) \in \ell(\mathfrak{r}) \Leftrightarrow \mathfrak{x}_{\mathfrak{r}}=\min \left(\mathfrak{x}_{\mathfrak{r}}^{1}, x_{\mathfrak{r}}^{2}\right)$, if $\left(\mathfrak{\jmath}, x_{\mathfrak{r}}^{1}\right) \in \Delta_{1}(\mathfrak{r}) \&\left(\mathfrak{\jmath}, \mathfrak{x}_{\mathfrak{r}}^{2}\right) \in \Delta_{2}(\mathfrak{r})$.

Definition 14: [30] Suppose $\Gamma_{1}=\left(\Delta_{1}, \Re_{1}, N_{1}\right)$ and $\Gamma_{2}=\left(\Delta_{2}, \Re_{2}, N_{2}\right)$ are two N-SSs, then their extended union and intersection are interpreted as:

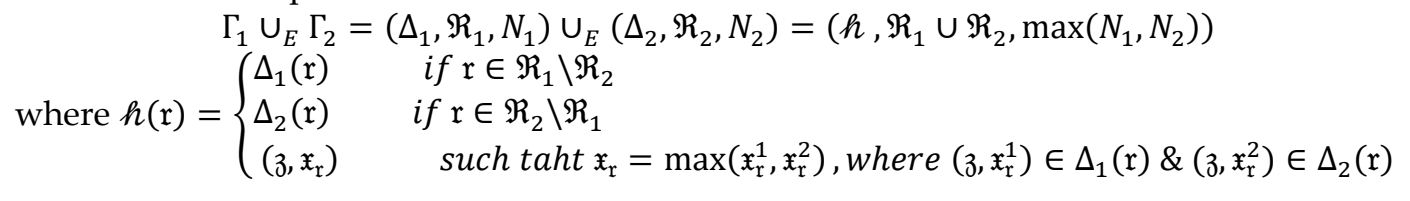

$$
\begin{aligned}
& \Gamma_{1} \cap_{E} \Gamma_{2}=\left(\Delta_{1}, \Re_{1}, N_{1}\right) \cap_{e}\left(\Delta_{2}, \Re_{2}, N_{2}\right)=\left(\ell, \Re_{1} \cup \Re_{2}, \max \left(N_{1}, N_{2}\right)\right) \\
& \text { where } \ell(\mathfrak{r})=\left\{\begin{array}{ll}
\Delta_{1}(\mathfrak{r}) & \text { if } \mathfrak{r} \in \mathfrak{R}_{1} \backslash \mathfrak{R}_{2} \\
\Delta_{2}(\mathfrak{r}) & \text { if } \mathfrak{r} \in \mathfrak{R}_{2} \backslash \mathfrak{R}_{1} \\
\left(\mathfrak{\jmath}, \mathfrak{x}_{\mathfrak{r}}\right) & \text { such that } \mathfrak{x}_{\mathfrak{r}}=\min \left(\mathfrak{x}_{\mathfrak{r}}^{1}, \mathfrak{x}_{\mathfrak{r}}^{2}\right), \text { where, }\left(\mathfrak{\jmath}, \mathfrak{x}_{\mathfrak{r}}^{1}\right) \in \Delta_{1}(\mathfrak{r}) \&\left(\mathfrak{z}, \mathfrak{x}_{\mathfrak{r}}^{2}\right) \in \Delta_{2}(\mathfrak{r})
\end{array}\right. \text {. }
\end{aligned}
$$

Definition 15: [37] Suppose a universal set $Z$, the set of attributes $\beta$, and $\mathfrak{X}=\{0,1,2, \ldots, N-1\}$ be an ordered grades set with $N \in\{2,3, \cdots\}$, then a pair $(\Omega,((\Delta, \Re, N)))$ is said to be IFN-SS where $(\Delta, \Re, N)$ is an N-SS on $\mathcal{Z}$, and $\Omega: \Re \rightarrow I F^{Z \times \mathfrak{X}}$, where $I F^{Z \times \mathfrak{X}}$ is the set of all IFS over $Z \times \mathfrak{X}$.

\section{A novel notion of complex picture fuzzy sets}

In this part of the article, we establish a novel definition of CPFSs which is the fusion of CFSs and PFSs. We also establish some properties like a complement, union, and intersection.

Definition 16: A CPFS $\mathcal{H}$ is elaborated by:

$$
\mathcal{H}=\left\{\left(z,\left(\phi_{\mathcal{H}}(z), \varphi_{\mathcal{H}}(z), \psi_{\mathcal{H}}(z)\right)\right): z \in Z\right\},
$$

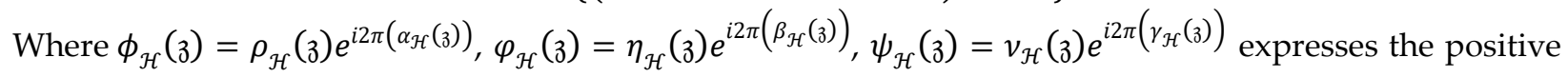
grade, neutral or abstain grade, and negative grade in the form of complex number with some rules that are $\rho_{\mathcal{H}}(\mathfrak{z}), \eta_{\mathcal{H}}(\mathfrak{z}), v_{\mathcal{H}}(\mathfrak{z}) \in[0,1]$ are the amplitude terms and $\alpha_{\mathcal{H}}(\mathfrak{z}), \beta_{\mathcal{H}}(\mathfrak{z}), \gamma_{\mathcal{H}}(\mathfrak{z}) \in[0,1]$ are the phase terms, respectively by using the conditions $0 \leq \rho_{\mathcal{H}}(3)+\eta_{\mathcal{H}}(3)+v_{\mathcal{H}}(3) \leq 1$ and $0 \leq \alpha_{\mathcal{H}}(3)+\beta_{\mathcal{H}}(3)+$ $\gamma_{\mathcal{H}}(3) \leq 1$ for all $\mathfrak{z} \in \mathcal{Z}$. The triplet $\mathcal{H}=\left(\phi_{\mathcal{H}}(3), \varphi_{\mathcal{H}}(3), \psi_{\mathcal{H}}(3)\right)$ expressed the complex PFN (CPFN).

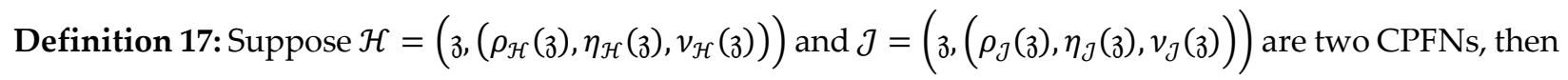

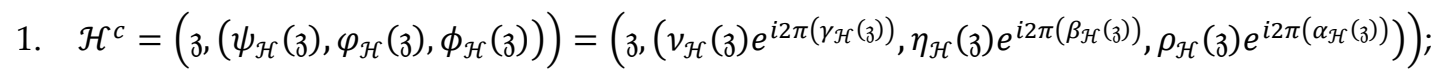

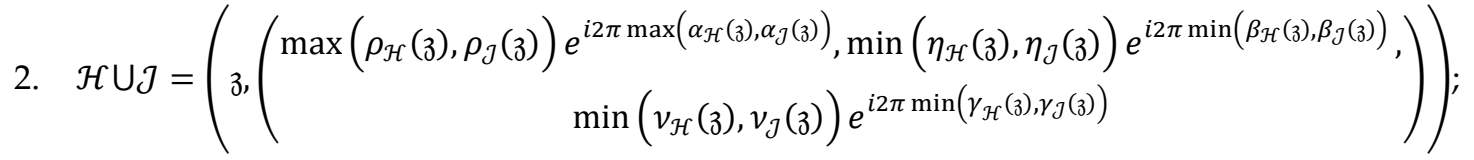

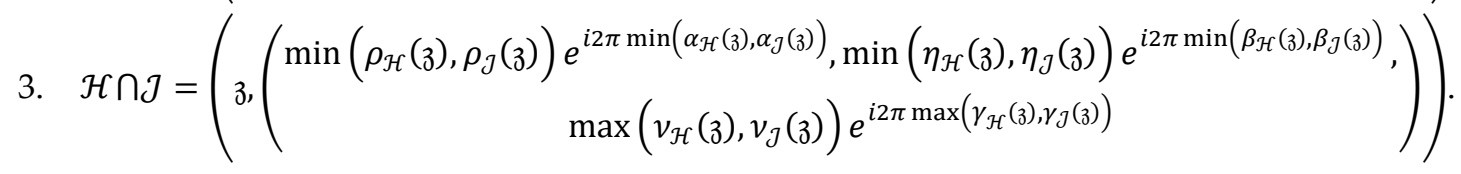

\section{The notion of complex picture fuzzy N-soft sets}


In this segment of the manuscript, we define the idea of CPFN-SS and also define some fundamental properties of CPFN-SS like a complement, intersection, union, etc. along with examples. Throughout this article, the universal set be designated by $Z$, the set of attributes be designated by $B$, and the set of ordered grades be designated by $\mathfrak{X}=\{0,1,2, \ldots, N-1\}$, and N-SS over $Z$ by $\Gamma=(\Delta, \Re, N)$, where $\Re \subseteq \Re$.

Definition 18: Suppose $Z$ is a universal set with $\Re \subseteq ß$ is a set of attributes, a pair $(\Psi, \Gamma)$ is called CPFN-SS over $Z$, where $\Gamma=(\Delta, \Re, N)$ be an N-SS over $Z$ with $N \in\{2,3, \ldots\}$ and $\Psi$ is a mapping from $\Re$ to $C P F(Z)$ i.e. $\Psi: \Re \rightarrow C P F(\mathcal{Z})$, where $C P F(\mathcal{Z})$ be the set of all CPFSs over $\mathcal{Z}$.

In the definition 18, we assign a CPFS on the image of the parameter under the mapping $\Delta$ by a mapping $\Psi$ to each parameter. Thus, for all $\mathfrak{r} \in \mathfrak{R}$ and $\mathfrak{z} \in \mathcal{Z} \exists$ a unique $\left(\mathfrak{z}, \mathfrak{x}_{\mathfrak{r}}\right) \in \mathcal{Z} \times \mathfrak{X}$ such that $\mathfrak{x}_{\mathfrak{r}} \in \mathfrak{X}$ and

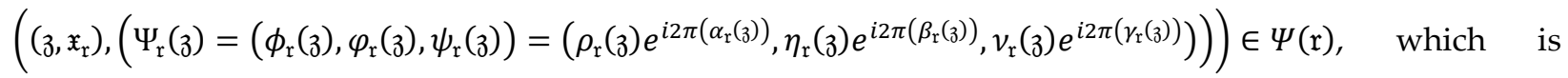
notation that summarize to $\Psi_{\mathfrak{r}}(\mathfrak{z})=\Psi(\mathfrak{r})\left(\mathfrak{z}, \mathfrak{x}_{\mathfrak{r}}\right)$.

Example 1: Suppose $Z=\left\{z_{1}, z_{2}, z_{3}, \jmath_{4}\right\}$ is a set of four alternatives and $\Re=\left\{\mathfrak{r}_{1}, \mathfrak{r}_{2}, \mathfrak{r}_{3}\right\}$ is a set of attributes. An expert allots grades to these alternatives based on attributes $\mathfrak{r}_{1}, \mathfrak{r}_{2}$ and $\mathfrak{r}_{3}$ in the shape of circle stars and circle hole which is represented in table 1, where Four circle stars is for "Excellent", three circle stars is for "very good", two circle stars is for "good", one circle star is for "normal", circle hole is for "poor".

Table 1. Information provided by an expert.

\begin{tabular}{|c|c|c|c|}
\hline$z$ & $r_{1}$ & $\mathfrak{r}_{2}$ & $\mathfrak{r}_{3}$ \\
\hline $\mathbf{z}_{1}$ & $\circledast \circledast$ & ○) & 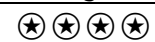 \\
\hline $3_{2}$ & 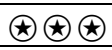 & $(\star)$ & () \\
\hline $3_{3}$ & $\circledast$ & 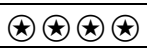 & $(\star)$ \\
\hline $3_{4}$ & 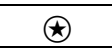 & $\circledast \otimes$ & $\oplus \circledast \circledast$ \\
\hline
\end{tabular}

We can link the set $\mathfrak{X}=\{0,1,2,3,4\}$ with the circle stars and circle hole displayed in table (1) by saying that 4 characterizes $" \circledast \circledast \circledast \circledast$ ", 3 characterizes $" \circledast \circledast \circledast$ ", 2 characterizes $" \circledast \circledast ", 1$ signifies $" \circledast "$, and 0 characterizes " $\odot "$ ". Then the 5-SS $(\Delta, \Re, 5)$ is defined as

$$
\begin{aligned}
& \Delta\left(\mathfrak{r}_{1}\right)=\left\{\left(\mathfrak{z}_{1}, 2\right),\left(\mathfrak{z}_{2}, 3\right),\left(3_{3}, 1\right),\left(z_{4}, 1\right)\right\} \\
& \Delta\left(\mathfrak{r}_{2}\right)=\left\{\left(3_{1}, 0\right),\left(z_{2}, 1\right),\left(z_{3}, 4\right),\left(z_{4}, 2\right)\right\} \\
& \Delta\left(\mathfrak{r}_{3}\right)=\left\{\left(3_{1}, 4\right),\left(3_{2}, 0\right),\left(3_{3}, 1\right),\left(3_{4}, 3\right)\right\}
\end{aligned}
$$

In table 1, we display the tabular form of 5-SS

Table 2. 5-SS in tabular form.

\begin{tabular}{|c|c|c|c|}
\hline$(\boldsymbol{\Delta}, \boldsymbol{R}, \mathbf{5})$ & $\mathfrak{r}_{\mathbf{1}}$ & $\mathfrak{r}_{\mathbf{2}}$ & $\mathfrak{r}_{\mathbf{3}}$ \\
\hline $\boldsymbol{3}_{1}$ & 2 & 0 & 4 \\
\hline $\boldsymbol{3}_{2}$ & 3 & 1 & 0 \\
\hline $\boldsymbol{3}_{3}$ & 1 & 4 & 1 \\
\hline $\boldsymbol{3}_{4}$ & 1 & 2 & 3 \\
\hline
\end{tabular}

$\phi, \varphi$, and $\psi$ will satisfy the following grading criteria. If grade is 0 , then $0.0 \leq \phi(3)<0.2$, (i.e. $0.0 \leq$ $\left.\frac{\rho(3)+\alpha(3)}{2} \leq 0.2\right)$ and $0 \leq \phi(3)+\varphi(3)+\psi(3) \leq 1$, i.e. $0 \leq \rho(3)+\eta(3)+v(z) \leq 1$ and $0 \leq \alpha(3)+\beta(3)+\gamma(3) \leq$ 
1 , where $0 \leq \rho(3), \eta(3), v(3), \alpha(3), \beta(3), \gamma(3) \leq 1$ for each $z \in Z$. In the similar manner, if grade is 1 then $0.2 \leq$ $\phi(3)<0.4$, if grade is 2 then $0.4 \leq \phi(3)<0.6$, if grade is 3 then $0.6 \leq \phi(3)<0.8$, and if grade is 4 then $0.8 \leq$ $\phi(3) \leq 1.0$. Then the CPF5-SS is established below:

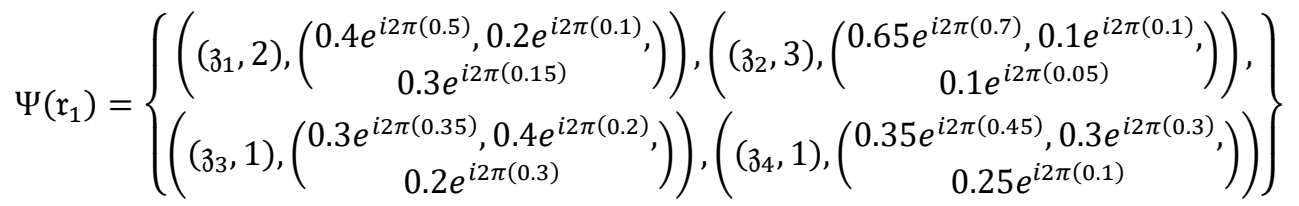

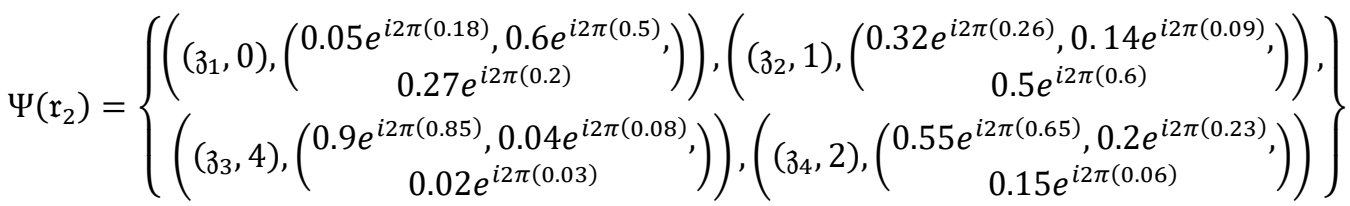

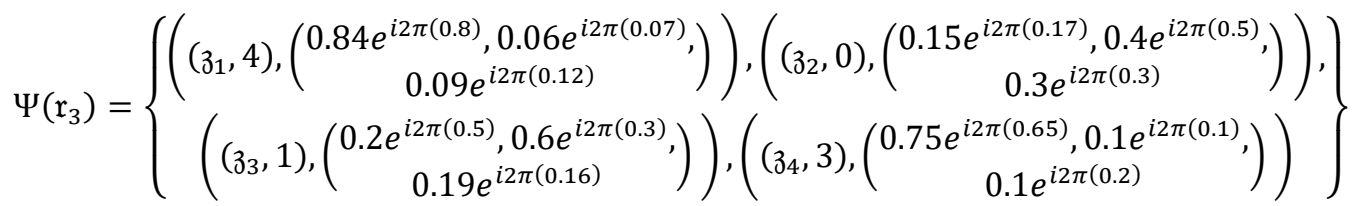

In table 3 we display the tabular form of CPF5-SS.

Table 3. The Tabular representation of CPF5-SS of example 1.

\begin{tabular}{|c|c|c|c|}
\hline$(\Psi,(\Delta, \mathfrak{R}, \mathbf{5}))$ & $\mathfrak{r}_{1}$ & $\mathfrak{r}_{2}$ & $\mathfrak{r}_{3}$ \\
\hline $\mathbf{z}_{1}$ & $2,\left(\begin{array}{c}0.4 e^{i 2 \pi(0.5)} \\
0.2 e^{i 2 \pi(0.1)} \\
0.3 e^{i 2 \pi(0.15)}\end{array}\right)$ & $0,\left(\begin{array}{c}0.05 e^{i 2 \pi(0.18)} \\
0.6 e^{i 2 \pi(0.5)}, \\
0.27 e^{i 2 \pi(0.2)}\end{array}\right)$ & $4,\left(\begin{array}{l}0.84 e^{i 2 \pi(0.8)}, \\
0.06 e^{i 2 \pi(0.07)} \\
0.09 e^{i 2 \pi(0.12)}\end{array}\right)$ \\
\hline 32 & $3,\left(\begin{array}{c}0.65 e^{i 2 \pi(0.7)} \\
0.1 e^{i 2 \pi(0.1)}, \\
0.1 e^{i 2 \pi(0.05)}\end{array}\right)$ & $1,\left(\begin{array}{c}0.32 e^{i 2 \pi(0.26)} \\
0.14 e^{i 2 \pi(0.09)} \\
0.5 e^{i 2 \pi(0.6)}\end{array}\right)$ & $0,\left(\begin{array}{c}0.15 e^{i 2 \pi(0.17)} \\
0.4 e^{i 2 \pi(0.5)} \\
0.3 e^{i 2 \pi(0.3)}\end{array}\right)$ \\
\hline 33 & $1,\left(\begin{array}{c}0.3 e^{i 2 \pi(0.35)} \\
0.4 e^{i 2 \pi(0.2)} \\
0.2 e^{i 2 \pi(0.3)}\end{array}\right)$ & $4,\left(\begin{array}{c}0.9 e^{i 2 \pi(0.85)} \\
0.04 e^{i 2 \pi(0.08)} \\
0.02 e^{i 2 \pi(0.03)}\end{array}\right)$ & $1,\left(\begin{array}{c}0.2 e^{i 2 \pi(0.5)}, \\
0.6 e^{i 2 \pi(0.3)} \\
0.19 e^{i 2 \pi(0.16)}\end{array}\right)$ \\
\hline 34 & $1,\left(\begin{array}{c}0.35 e^{i 2 \pi(0.45)} \\
0.3 e^{i 2 \pi(0.3)} \\
0.25 e^{i 2 \pi(0.1)}\end{array}\right)$ & $2,\left(\begin{array}{c}0.55 e^{i 2 \pi(0.65)} \\
0.2 e^{i 2 \pi(0.23)} \\
0.15 e^{i 2 \pi(0.06)}\end{array}\right)$ & $3,\left(\begin{array}{c}0.75 e^{i 2 \pi(0.65)} \\
0.1 e^{i 2 \pi(0.1)} \\
0.1 e^{i 2 \pi(0.2)}\end{array}\right)$ \\
\hline
\end{tabular}

Every CPFN-SS can be express as $\mathrm{CPF}(\mathrm{N}+1)-\mathrm{SS}$. For instance in example 1 we have CPF5-SS but we can also call it CPF6-SS.

Definition 19: A CPFN-SS $(\Psi, \Gamma=(\Delta, \Re, N))$ over $Z$ is called an efficient if for some $z_{j} \in \mathcal{Z}, \mathfrak{r}_{k} \in \Re$ we have $\Psi\left(\mathfrak{r}_{k}\right)=\left(\left(\jmath_{j}, N-1\right), 1.0 e^{i 2 \pi(1.0)}, 0.0 e^{i 2 \pi(0.0)}, 0.0 e^{i 2 \pi(0.0)}\right)$.

Example 2: Suppose a CPF5-SS over $Z$ in tabular representation which is demonstrate in table 4. This CPF5SS is efficient because for $z_{1} \in Z$ and $\mathfrak{r}_{3} \in \mathfrak{R}$ we have $\left(4,1.0 e^{i 2 \pi(1.0)}, 0.0 e^{i 2 \pi(0.0)}, 0.0 e^{i 2 \pi(0.0)}\right)$.

Table 4. The tabular form of the CPF5-SS presented in example 2.

\begin{tabular}{|c|c|c|c|}
\hline$(\Psi,(\Delta, \mathfrak{R}, \mathbf{5}))$ & $\mathfrak{r}_{1}$ & $\mathfrak{r}_{2}$ & $\mathfrak{r}_{3}$ \\
\hline
\end{tabular}




\begin{tabular}{|c|c|c|c|}
\hline 31 & $0,\left(\begin{array}{c}0.05 e^{i 2 \pi(0.18)} \\
0.6 e^{i 2 \pi(0.5)}, \\
0.27 e^{i 2 \pi(0.2)}\end{array}\right)$ & $2,\left(\begin{array}{c}0.4 e^{i 2 \pi(0.5)}, \\
0.2 e^{i 2 \pi(0.1)}, \\
0.3 e^{i 2 \pi(0.15)}\end{array}\right)$ & $4,\left(\begin{array}{c}1.0 e^{i 2 \pi(1.0)}, \\
0.0 e^{i 2 \pi(0.0)}, \\
0.0 e^{i 2 \pi(0.0)}\end{array}\right)$ \\
\hline 32 & $1,\left(\begin{array}{c}0.32 e^{i 2 \pi(0.26)}, \\
0.14 e^{i 2 \pi(0.09)}, \\
0.5 e^{i 2 \pi(0.6)}\end{array}\right)$ & $3,\left(\begin{array}{c}0.65 e^{i 2 \pi(0.7)}, \\
0.1 e^{i 2 \pi(0.1)}, \\
0.1 e^{i 2 \pi(0.05)}\end{array}\right)$ & $0,\left(\begin{array}{c}0.15 e^{i 2 \pi(0.17)} \\
0.4 e^{i 2 \pi(0.5)} \\
0.3 e^{i 2 \pi(0.3)}\end{array}\right)$ \\
\hline 33 & $1,\left(\begin{array}{c}0.3 e^{i 2 \pi(0.35)}, \\
0.4 e^{i 2 \pi(0.2)}, \\
0.2 e^{i 2 \pi(0.3)}\end{array}\right)$ & $4,\left(\begin{array}{c}0.9 e^{i 2 \pi(0.85)}, \\
0.04 e^{i 2 \pi(0.08)}, \\
0.02 e^{i 2 \pi(0.03)}\end{array}\right)$ & $1,\left(\begin{array}{c}0.2 e^{i 2 \pi(0.5)}, \\
0.6 e^{i 2 \pi(0.3)}, \\
0.19 e^{i 2 \pi(0.16)}\end{array}\right)$ \\
\hline 34 & $1,\left(\begin{array}{c}0.35 e^{i 2 \pi(0.45)} \\
0.3 e^{i 2 \pi(0.3)} \\
0.25 e^{i 2 \pi(0.1)}\end{array}\right)$ & $2,\left(\begin{array}{c}0.55 e^{i 2 \pi(0.65)} \\
0.2 e^{i 2 \pi(0.23)}, \\
0.15 e^{i 2 \pi(0.06)}\end{array}\right)$ & $3,\left(\begin{array}{c}0.75 e^{i 2 \pi(0.65)} \\
0.1 e^{i 2 \pi(0.1)}, \\
0.1 e^{i 2 \pi(0.2)}\end{array}\right)$ \\
\hline
\end{tabular}

Definition 20: An efficient CPFN-SS $(\Psi, \Gamma=(\Delta, \Re, N))$ is called minimized efficient signified by $\left(\Psi_{m}, \Gamma_{m}=\left(\Delta_{m}, \Re, N\right)\right)$ and illustrate as $m=\max \Delta_{m}\left(\mathfrak{r}_{k}\right)\left(\jmath_{j}\right)+1,\left(1.0 e^{i 2 \pi(1.0)}, 0.0 e^{i 2 \pi(0.0)}, 0.0 e^{i 2 \pi(0.0)}\right)$ for some $z_{j}$ and $\Delta_{m}\left(\mathfrak{r}_{k}\right)\left(\mathfrak{z}_{j}\right)=\Delta\left(\mathfrak{r}_{k}\right)\left(\mathfrak{z}_{j}\right)$ for all $\mathfrak{r}_{k} \in \mathfrak{R}, \mathfrak{z}_{j} \in \mathcal{Z}$.

Definition 21: Suppose a CPFN-SS $(\Psi, \Gamma=(\Delta, \Re, N))$ on $Z$, then the CPF weak complement of the CPFNSS is signified by $\left(\Psi^{c}, \Gamma^{c}=\left(\Delta^{c}, \Re, N\right)\right)$, where $\left(\Delta^{c}, \Re, N\right)$ is weak complement of N-SS that is, $\Delta\left(\mathfrak{r}_{k}\right) \cap \Delta^{c}\left(\mathfrak{r}_{k}\right)=$ $\emptyset \forall \mathfrak{r}_{k} \in \mathfrak{R}$, and $\Psi^{c}\left(\mathfrak{r}_{k}\right)=\left\{\left(\mathfrak{z}_{j}, \mathfrak{x}_{\mathfrak{r}_{k}}\right),\left(\psi\left(\mathfrak{z}_{j}, \mathfrak{x}_{\mathfrak{r}_{k}}\right), \varphi\left(\mathfrak{z}_{j}, \mathfrak{x}_{\mathfrak{r}_{k}}\right), \phi\left(\mathfrak{z}_{j}, \mathfrak{x}_{\mathfrak{r}_{k}}\right)\right) \mid\left(\mathfrak{z}_{j}, \mathfrak{x}_{\mathfrak{r}_{k}}\right) \in Z \times \mathfrak{X}\right\} \forall \mathfrak{r}_{k} \in \Re$.

Example 3: Suppose a CPF5-SS $(\Psi, \Gamma=(\Delta, \Re, 5))$ of example 1, then the CPF weak complement of CPF5-SS is demonstrated in tabular representation in table 5

Table 5. The tabular form of CPF week complement of CPF5-SS of example 1.

\begin{tabular}{|c|c|c|c|}
\hline$\left(\Psi^{c},\left(\Delta^{c}, \mathfrak{R}, 5\right)\right)$ & $\mathfrak{r}_{1}$ & $\mathfrak{r}_{2}$ & $\mathfrak{r}_{3}$ \\
\hline 31 & $4,\left(\begin{array}{c}0.3 e^{i 2 \pi(0.15)} \\
0.2 e^{i 2 \pi(0.1)}, \\
0.4 e^{i 2 \pi(0.5)}\end{array}\right)$ & $3,\left(\begin{array}{c}0.27 e^{i 2 \pi(0.2)} \\
0.6 e^{i 2 \pi(0.5)} \\
0.05 e^{i 2 \pi(0.18)}\end{array}\right)$ & $2,\left(\begin{array}{c}0.09 e^{i 2 \pi(0.12)} \\
0.06 e^{i 2 \pi(0.07)} \\
0.84 e^{i 2 \pi(0.8)}\end{array}\right)$ \\
\hline 32 & $4,\left(\begin{array}{c}0.1 e^{i 2 \pi(0.05)} \\
0.1 e^{i 2 \pi(0.1)} \\
0.65 e^{i 2 \pi(0.7)}\end{array}\right)$ & $3,\left(\begin{array}{c}0.5 e^{i 2 \pi(0.6)}, \\
0.14 e^{i 2 \pi(0.09)} \\
0.32 e^{i 2 \pi(0.26)}\end{array}\right)$ & $2,\left(\begin{array}{c}0.3 e^{i 2 \pi(0.3)} \\
0.4 e^{i 2 \pi(0.5)} \\
0.15 e^{i 2 \pi(0.17)}\end{array}\right)$ \\
\hline 33 & $0,\left(\begin{array}{c}0.2 e^{i 2 \pi(0.3)}, \\
0.4 e^{i 2 \pi(0.2)}, \\
0.3 e^{i 2 \pi(0.35)}\end{array}\right)$ & $3,\left(\begin{array}{c}0.02 e^{i 2 \pi(0.03)} \\
0.04 e^{i 2 \pi(0.08)} \\
0.9 e^{i 2 \pi(0.85)}\end{array}\right)$ & $2,\left(\begin{array}{c}0.19 e^{i 2 \pi(0.16)} \\
0.6 e^{i 2 \pi(0.3)} \\
0.2 e^{i 2 \pi(0.5)}\end{array}\right)$ \\
\hline 34 & $0,\left(\begin{array}{c}0.25 e^{i 2 \pi(0.1)} \\
0.3 e^{i 2 \pi(0.3)} \\
0.35 e^{i 2 \pi(0.45)}\end{array}\right)$ & $3,\left(\begin{array}{c}0.15 e^{i 2 \pi(0.06)} \\
0.2 e^{i 2 \pi(0.23)} \\
0.55 e^{i 2 \pi(0.65)}\end{array}\right)$ & $2,\left(\begin{array}{c}0.1 e^{i 2 \pi(0.2)}, \\
0.1 e^{i 2 \pi(0.1)} \\
0.75 e^{i 2 \pi(0.65)}\end{array}\right)$ \\
\hline
\end{tabular}

Definition 22: For a CPFN-SS $(\Psi, \Gamma=(\Delta, \Re, N))$, the top CPF weak complement of $(\Psi, \Gamma=(\Delta, \Re, N))$ is $\left(\Psi^{c}, \Gamma^{T}=\left(\Delta^{T}, \Re, N\right)\right)$ where,

$$
\Delta^{T}\left(\mathfrak{r}_{k}\right)= \begin{cases}N-1 & \text { if } \mathfrak{x}_{\mathfrak{r}_{k}}<N-1 \\ 0 & \text { if } \mathfrak{x}_{\mathfrak{r}_{k}}=N-1\end{cases}
$$

Example 23: Suppose a CPF5-SS $(\Psi, \Gamma=(\Delta, \Re, 5))$ of example 1, then the top CPF weak complement of CPF5-SS is demonstrated in tabular representation in table 6. 
Table 6. The tabular representation of top CPF week complement of CPF5-SS of example 1.

\begin{tabular}{|c|c|c|c|}
\hline$\left(\boldsymbol{\Psi}^{\boldsymbol{c}},\left(\boldsymbol{\Delta}^{\boldsymbol{T}}, \boldsymbol{R}, \mathbf{5}\right)\right)$ & $\mathfrak{r}_{\mathbf{1}}$ & $\mathfrak{r}_{2}$ & $\mathfrak{r}_{3}$ \\
\hline $\mathbf{3}_{1}$ & $4,\left(\begin{array}{c}0.3 e^{i 2 \pi(0.15)}, \\
0.2 e^{i 2 \pi(0.1)}, \\
0.4 e^{i 2 \pi(0.5)}\end{array}\right)$ & $4,\left(\begin{array}{c}0.27 e^{i 2 \pi(0.2)}, \\
0.6 e^{i 2 \pi(0.5)}, \\
0.05 e^{i 2 \pi(0.18)}\end{array}\right)$ & $0,\left(\begin{array}{c}0.09 e^{i 2 \pi(0.12)}, \\
0.06 e^{i 2 \pi(0.07)} \\
0.84 e^{i 2 \pi(0.8)}\end{array}\right)$ \\
\hline $\mathbf{3}_{2}$ & $4,\left(\begin{array}{c}0.1 e^{i 2 \pi(0.05)}, \\
0.1 e^{i 2 \pi(0.1)}, \\
0.65 e^{i 2 \pi(0.7)}\end{array}\right)$ & $4,\left(\begin{array}{c}0.5 e^{i 2 \pi(0.6)}, \\
0.14 e^{i 2 \pi(0.09)}, \\
0.32 e^{i 2 \pi(0.26)}\end{array}\right)$ & $4,\left(\begin{array}{c}0.3 e^{i 2 \pi(0.3)}, \\
0.4 e^{i 2 \pi(0.5)}, \\
0.15 e^{i 2 \pi(0.17)}\end{array}\right)$ \\
\hline $3_{3}$ & $4,\left(\begin{array}{c}0.2 e^{i 2 \pi(0.3)}, \\
0.4 e^{i 2 \pi(0.2)}, \\
0.3 e^{i 2 \pi(0.35)}\end{array}\right)$ & $0,\left(\begin{array}{c}0.02 e^{i 2 \pi(0.03)}, \\
0.04 e^{i 2 \pi(0.08)} \\
0.9 e^{i 2 \pi(0.85)}\end{array}\right)$ & $4,\left(\begin{array}{c}0.19 e^{i 2 \pi(0.16)} \\
0.6 e^{i 2 \pi(0.3)}, \\
0.2 e^{i 2 \pi(0.5)}\end{array}\right)$ \\
\hline $\mathbf{3}_{4}$ & $4,\left(\begin{array}{c}0.25 e^{i 2 \pi(0.1)}, \\
0.3 e^{i 2 \pi(0.3)} \\
0.35 e^{i 2 \pi(0.45)}\end{array}\right)$ & $4,\left(\begin{array}{c}0.15 e^{i 2 \pi(0.06)} \\
0.2 e^{i 2 \pi(0.23)}, \\
0.55 e^{i 2 \pi(0.65)}\end{array}\right)$ & $4,\left(\begin{array}{c}0.1 e^{i 2 \pi(0.2)}, \\
0.1 e^{i 2 \pi(0.1)}, \\
0.75 e^{i 2 \pi(0.65)}\end{array}\right)$ \\
\hline
\end{tabular}

Definition 24: For a CPFN-SS $(\Psi, \Gamma=(\Delta, \Re, N))$, the bottom CPF weak complement of a CPFN-SS $(\Psi, \Gamma=(\Delta, \Re, 5))$ is $\left(\Psi^{c}, \Gamma^{B}=\left(\Delta^{B}, \Re, 5\right)\right)$ where,

$$
\Delta^{B}\left(\mathfrak{r}_{k}\right)= \begin{cases}0 & \text { if } \mathfrak{x}_{\mathfrak{r}_{k}}>0 \\ N-1 & \text { if } \mathfrak{x}_{\mathfrak{r}_{k}}=0\end{cases}
$$

Example 5: Suppose a CPF5-SS $(\Psi, \Gamma=(\Delta, \Re, 5))$ of example 1, then the bottom CPF weak complement of CPF5-SS is demonstrated in tabular representation in table 7.

Table 7. The tabular representation of bottom CPF week complement of CPF5-SS of example 1.

\begin{tabular}{|c|c|c|c|}
\hline$\left(\Psi^{c},\left(\Delta^{B}, \mathfrak{R}, \mathbf{5}\right)\right)$ & $\mathbf{r}_{1}$ & $\mathfrak{r}_{2}$ & $\mathbf{r}_{3}$ \\
\hline $3_{1}$ & $0,\left(\begin{array}{c}0.3 e^{i 2 \pi(0.15)} \\
0.2 e^{i 2 \pi(0.1)} \\
0.4 e^{i 2 \pi(0.5)}\end{array}\right)$ & $4,\left(\begin{array}{c}0.27 e^{i 2 \pi(0.2)}, \\
0.6 e^{i 2 \pi(0.5)}, \\
0.05 e^{i 2 \pi(0.18)}\end{array}\right)$ & $0,\left(\begin{array}{c}0.09 e^{i 2 \pi(0.12)}, \\
0.06 e^{i 2 \pi(0.07)} \\
0.84 e^{i 2 \pi(0.8)}\end{array}\right)$ \\
\hline 32 & $0,\left(\begin{array}{c}0.1 e^{i 2 \pi(0.05)} \\
0.1 e^{i 2 \pi(0.1)} \\
0.65 e^{i 2 \pi(0.7)}\end{array}\right)$ & $0,\left(\begin{array}{c}0.5 e^{i 2 \pi(0.6)}, \\
0.14 e^{i 2 \pi(0.09)} \\
0.32 e^{i 2 \pi(0.26)}\end{array}\right)$ & $4,\left(\begin{array}{c}0.3 e^{i 2 \pi(0.3)}, \\
0.4 e^{i 2 \pi(0.5)} \\
0.15 e^{i 2 \pi(0.17)}\end{array}\right)$ \\
\hline 33 & $0,\left(\begin{array}{c}0.2 e^{i 2 \pi(0.3)}, \\
0.4 e^{i 2 \pi(0.2)}, \\
0.3 e^{i 2 \pi(0.35)}\end{array}\right)$ & $0,\left(\begin{array}{c}0.02 e^{i 2 \pi(0.03)} \\
, 0.04 e^{i 2 \pi(0.08)} \\
0.9 e^{i 2 \pi(0.85)}\end{array}\right)$ & $0,\left(\begin{array}{c}0.19 e^{i 2 \pi(0.16)}, \\
0.6 e^{i 2 \pi(0.3)}, \\
0.2 e^{i 2 \pi(0.5)}\end{array}\right.$ \\
\hline 34 & $0,\left(\begin{array}{c}0.25 e^{i 2 \pi(0.1)} \\
0.3 e^{i 2 \pi(0.3)} \\
0.35 e^{i 2 \pi(0.45)}\end{array}\right)$ & $0,\left(\begin{array}{c}0.15 e^{i 2 \pi(0.06)} \\
0.2 e^{i 2 \pi(0.23)} \\
0.55 e^{i 2 \pi(0.65)}\end{array}\right)$ & $0,\left(\begin{array}{c}0.1 e^{i 2 \pi(0.2)}, \\
0.1 e^{i 2 \pi(0.1)}, \\
0.75 e^{i 2 \pi(0.65)}\end{array}\right)$ \\
\hline
\end{tabular}

Definition 25: Suppose $\left(\Psi_{1}, \Gamma_{1}=\left(\Delta_{1}, \Re_{1}, N_{1}\right)\right)$ and $\left(\Psi_{2}, \Gamma_{2}=\left(\Delta_{2}, \Re_{2}, N_{2}\right)\right)$ are two CPFN-SS over $Z$, then their restricted intersection is signified by $\left(\Psi_{1}, \Gamma_{1}=\left(\Delta_{1}, \Re_{1}, N_{1}\right)\right) \cap_{\mathcal{R}}\left(\Psi_{2}, \Gamma_{2}=\left(\Delta_{2}, \Re_{2}, N_{2}\right)\right)$ and is define as $\left(\Omega, \Gamma_{1} \cap_{\mathcal{R}} \Gamma_{2}\right)$, where $\Gamma_{1} \cap_{\mathcal{R}} \Gamma_{2}=\left(\partial, \Re_{1} \cap \Re_{2}, \min \left(N_{1}, N_{2}\right)\right) \forall \mathfrak{r}_{k} \in \Re_{1} \cap \Re_{2}, \jmath_{j} \in Z,\left(\left(\jmath_{j}, \mathfrak{x}_{\mathfrak{r}_{k}}\right), \sigma, \varrho, \tau\right) \in \Omega\left(\mathfrak{r}_{k}\right) \Leftrightarrow$ $\mathfrak{x}_{\mathfrak{r}_{k}}=\min \left(\mathfrak{x}_{\mathfrak{r}_{k}}^{1}, \mathfrak{x}_{\mathfrak{r}_{k}}^{2}\right), \sigma=\min \left(\phi_{1}\left(\jmath_{j}, \mathfrak{x}_{\mathfrak{r}_{k}}^{1}\right), \phi_{2}\left(\jmath_{j}, x_{\mathfrak{r}_{k}}^{2}\right)\right)=\left(\min \left(\rho_{1}\left(\jmath_{j}, \mathfrak{x}_{\mathfrak{r}_{k}}^{1}\right), \rho_{2}\left(\jmath_{j}, \mathfrak{x}_{\mathfrak{r}_{k}}^{2}\right)\right) e^{i 2 \pi \min \left(\alpha_{1}\left(\jmath_{j}, x_{\mathfrak{x}_{k}}^{1}\right), \alpha_{2}\left(\mathfrak{z}_{j}, \mathfrak{x}_{\mathfrak{r}_{k}}^{2}\right)\right)}\right)$, $\varrho=\min \left(\varphi_{1}\left(\jmath_{j}, x_{\mathfrak{r}_{k}}^{1}\right), \varphi_{2}\left(\jmath_{j}, \mathfrak{x}_{\mathfrak{r}_{k}}^{2}\right)\right)=\left(\min \left(\eta_{1}\left(\jmath_{j}, x_{\mathfrak{x}_{k}}^{1}\right), \eta_{2}\left(\jmath_{j}, \mathfrak{x}_{\mathfrak{r}_{k}}^{2}\right)\right) e^{i 2 \pi \min \left(\beta_{1}\left(\jmath_{j}, x_{\mathfrak{x}_{k}}^{1}\right), \beta_{2}\left(\jmath_{j}, x_{\mathfrak{r}_{k}}^{2}\right)\right)}\right), \quad$ and $\quad \tau=$ 
$\max \left(\psi_{1}\left(\jmath_{j}, \mathfrak{x}_{\mathfrak{r}_{k}}^{1}\right), \psi_{2}\left(\mathfrak{z}_{j}, \mathfrak{x}_{\mathfrak{r}_{k}}^{2}\right)\right)=\left(\max \left(v_{1}\left(\mathfrak{z}_{j}, \mathfrak{x}_{\mathfrak{r}_{k}}^{1}\right), v_{2}\left(\mathfrak{z}_{j}, \mathfrak{x}_{\mathfrak{r}_{k}}^{2}\right)\right) e^{i 2 \pi \max \left(\gamma_{1}\left(z_{j}, x_{\mathfrak{x}_{k}}^{1}\right), \gamma_{2}\left(z_{j}, x_{\mathfrak{r}_{k}}^{2}\right)\right)}\right)$

$\left(\left(\jmath_{j}, x_{\mathfrak{r}_{k}}^{1}\right), \phi_{1}\left(\jmath_{j}, x_{\mathfrak{r}_{k}}^{1}\right), \varphi_{1}\left(\jmath_{j}, x_{\mathfrak{r}_{k}}^{1}\right), \psi_{1}\left(\jmath_{j}, \mathfrak{x}_{\mathfrak{r}_{k}}^{1}\right)\right) \in \Psi_{1}\left(\mathfrak{r}_{k}\right)$ and $\left(\left(\jmath_{j}, x_{\mathfrak{r}_{k}}^{2}\right), \phi_{2}\left(\jmath_{j}, x_{\mathfrak{r}_{k}}^{2}\right), \varphi_{2}\left(\jmath_{j}, x_{\mathfrak{r}_{k}}^{2}\right), \psi_{2}\left(\jmath_{j}, x_{\mathfrak{r}_{k}}^{2}\right)\right) \in \Psi_{2}\left(\mathfrak{r}_{k}\right)$.

Example 6: Suppose $Z=\left\{\mathfrak{z}_{1}, \mathfrak{z}_{2}, \mathfrak{z}_{3}\right\}$ is a universal set and $\mathfrak{R}_{1}, \mathfrak{R}_{2} \subseteq \Re$, where $\mathfrak{R}_{1}=\left\{\mathfrak{r}_{1}, \mathfrak{r}_{2}, \mathfrak{r}_{3}\right\}$, and $\mathfrak{R}_{2}=$ $\left\{\mathfrak{r}_{1}, \mathfrak{r}_{2}, \mathfrak{r}_{4}\right\}$. Suppose $\left(\Psi_{1}, \Gamma_{1}=\left(\Delta_{1}, \Re_{1}, 5\right)\right)$ and $\left(\Psi_{2}, \Gamma_{2}=\left(\Delta_{2}, \Re_{2}, 4\right)\right)$ are CPF5-SS and CPF4-SS over $Z$ respectively. The tabular representation of CPF5-SS $\left(\Psi_{1}, \Gamma_{1}=\left(\Delta_{1}, \Re_{1}, 5\right)\right)$ is demonstrated in table 8 and tabular representation of CPF4-SS $\left(\Psi_{2}, \Gamma_{2}=\left(\Delta_{2}, \Re_{2}, 4\right)\right)$ is demonstrated in table 9 . Then their restricted intersection is demonstrated in table 10.

Table 8. The CPF5-SS of example 6.

\begin{tabular}{|c|c|c|c|}
\hline$(\boldsymbol{\Psi},(\boldsymbol{\Delta}, \mathfrak{R}, \mathbf{5}))$ & $\mathfrak{r}_{\mathbf{1}}$ & $\mathfrak{r}_{2}$ & $\mathfrak{r}_{3}$ \\
\hline $\mathbf{3}_{1}$ & $2,\left(\begin{array}{c}0.4 e^{i 2 \pi(0.5)}, \\
0.2 e^{i 2 \pi(0.1)}, \\
0.3 e^{i 2 \pi(0.15)}\end{array}\right)$ & $0,\left(\begin{array}{c}0.05 e^{i 2 \pi(0.18)} \\
0.6 e^{i 2 \pi(0.5)}, \\
0.27 e^{i 2 \pi(0.2)}\end{array}\right)$ & $4,\left(\begin{array}{c}0.84 e^{i 2 \pi(0.8)}, \\
0.06 e^{i 2 \pi(0.07)} \\
0.09 e^{i 2 \pi(0.12)}\end{array}\right)$ \\
\hline $\mathbf{3}_{2}$ & $3,\left(\begin{array}{c}0.65 e^{i 2 \pi(0.7)}, \\
0.1 e^{i 2 \pi(0.1)}, \\
0.1 e^{i 2 \pi(0.05)}\end{array}\right)$ & $1,\left(\begin{array}{c}0.32 e^{i 2 \pi(0.26)}, \\
0.14 e^{i 2 \pi(0.09)}, \\
0.5 e^{i 2 \pi(0.6)}\end{array}\right)$ & $0,\left(\begin{array}{c}0.15 e^{i 2 \pi(0.17)}, \\
0.4 e^{i 2 \pi(0.5)}, \\
0.3 e^{i 2 \pi(0.3)}\end{array}\right)$ \\
\hline $3_{3}$ & $1,\left(\begin{array}{c}0.3 e^{i 2 \pi(0.35)}, \\
0.4 e^{i 2 \pi(0.2)}, \\
0.2 e^{i 2 \pi(0.3)}\end{array}\right)$ & $4,\left(\begin{array}{c}0.9 e^{i 2 \pi(0.85)}, \\
0.04 e^{i 2 \pi(0.08)}, \\
0.02 e^{i 2 \pi(0.03)}\end{array}\right)$ & $1,\left(\begin{array}{c}0.2 e^{i 2 \pi(0.5)}, \\
0.6 e^{i 2 \pi(0.3)}, \\
0.19 e^{i 2 \pi(0.16)}\end{array}\right)$ \\
\hline
\end{tabular}

Table 9. The CPF4-SS of example 6.

\begin{tabular}{|c|c|c|c|}
\hline$(\boldsymbol{\Psi},(\boldsymbol{\Delta}, \mathfrak{R}, \mathbf{4}))$ & $\mathfrak{r}_{\mathbf{1}}$ & $\mathfrak{r}_{2}$ & $\mathfrak{r}_{4}$ \\
\hline 31 & $1,\left(\begin{array}{c}0.5 e^{i 2 \pi(0.4)}, \\
0.1 e^{i 2 \pi(0.3)}, \\
0.2 e^{i 2 \pi(0.1)}\end{array}\right)$ & $0,\left(\begin{array}{c}0.15 e^{i 2 \pi(0.2)}, \\
0.5 e^{i 2 \pi(0.4)}, \\
0.2 e^{i 2 \pi(0.3)}\end{array}\right)$ & $2,\left(\begin{array}{c}0.74 e^{i 2 \pi(0.6)}, \\
0.11 e^{i 2 \pi(0.2)} \\
0.13 e^{i 2 \pi(0.1)}\end{array}\right)$ \\
\hline 32 & $2,\left(\begin{array}{c}0.69 e^{i 2 \pi(0.5)}, \\
0.1 e^{i 2 \pi(0.3)}, \\
0.18 e^{i 2 \pi(0.15)}\end{array}\right)$ & $0,\left(\begin{array}{c}0.2 e^{i 2 \pi(0.15)}, \\
0.3 e^{i 2 \pi(0.3)}, \\
0.4 e^{i 2 \pi(0.35)}\end{array}\right)$ & $1,\left(\begin{array}{c}0.5 e^{i 2 \pi(0.47)}, \\
0.2 e^{i 2 \pi(0.1)}, \\
0.2 e^{i 2 \pi(0.3)}\end{array}\right)$ \\
\hline 33 & $3,\left(\begin{array}{c}0.8 e^{i 2 \pi(0.8)}, \\
0.1 e^{i 2 \pi(0.1)}, \\
0.05 e^{i 2 \pi(0.05)}\end{array}\right)$ & $3,\left(\begin{array}{c}0.9 e^{i 2 \pi(0.85)}, \\
0.04 e^{i 2 \pi(0.08)}, \\
0.02 e^{i 2 \pi(0.03)}\end{array}\right)$ & $2,\left(\begin{array}{c}0.6 e^{i 2 \pi(0.57)} \\
0.3 e^{i 2 \pi(0.2)}, \\
0.09 e^{i 2 \pi(0.1)}\end{array}\right)$ \\
\hline
\end{tabular}

Table 10. The restricted intersection of CPF5-SS and CPF4-SS of example 6.

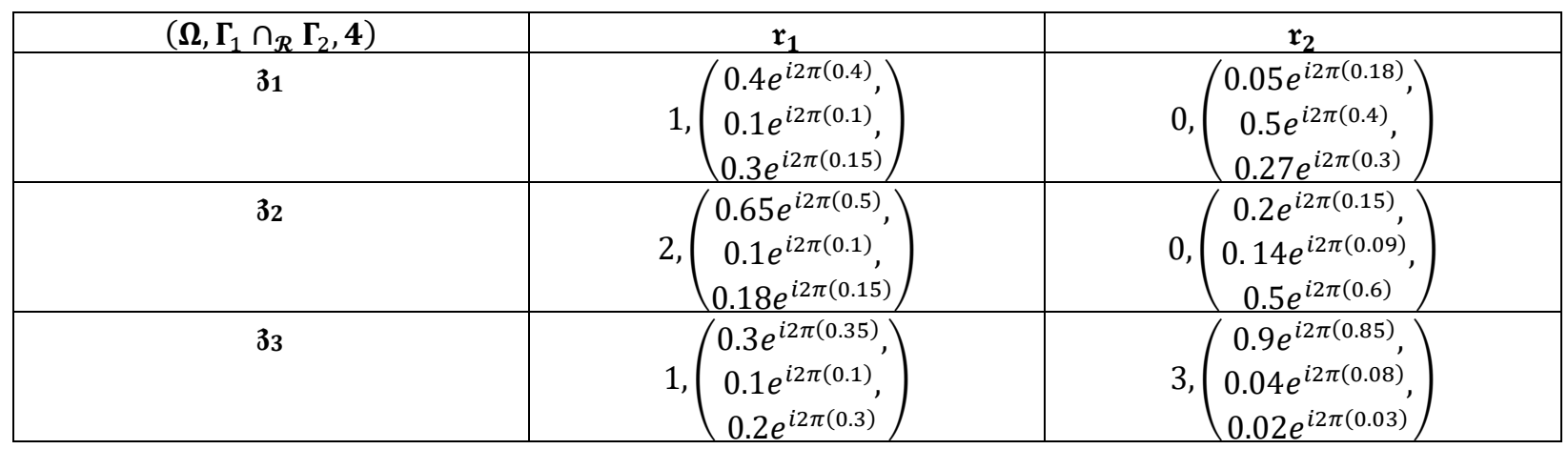


Definition 26: Suppose $\left(\Psi_{1}, \Gamma_{1}=\left(\Delta_{1}, \Re_{1}, N_{1}\right)\right)$ and $\left(\Psi_{2}, \Gamma_{2}=\left(\Delta_{2}, \Re_{2}, N_{2}\right)\right)$ are two CPFN-SS over $Z$, then their restricted union is signified by $\left(\Psi_{1}, \Gamma_{1}=\left(\Delta_{1}, \Re_{1}, N_{1}\right)\right) \cup_{\mathcal{R}}\left(\Psi_{2}, \Gamma_{2}=\left(\Delta_{2}, \Re_{2}, N_{2}\right)\right)$ and is define as $\left(\mho, \Gamma_{1} \cup_{\mathcal{R}} \Gamma_{2}\right)$, where $\Gamma_{1} \cup_{\mathcal{R}} \Gamma_{2}=\left(\mathcal{E}, \Re_{1} \cap \Re_{2}, \max \left(N_{1}, N_{2}\right)\right) \forall \mathfrak{r}_{k} \in \Re_{1} \cap \Re_{2}, \jmath_{j} \in \mathcal{Z},\left(\left(\jmath_{j}, \mathfrak{x}_{\mathfrak{r}_{k}}\right), \sigma, \varrho, \tau\right) \in \mho\left(\mathfrak{r}_{k}\right) \Leftrightarrow$ $\mathfrak{x}_{\mathfrak{r}_{k}}=\max \left(\mathfrak{x}_{\mathfrak{r}_{k}}^{1}, \mathfrak{x}_{\mathfrak{r}_{k}}^{2}\right)$, $\left(\max \left(\rho_{1}\left(\jmath_{j}, \mathfrak{x}_{\mathfrak{r}_{k}}^{1}\right), \rho_{2}\left(\jmath_{j}, \mathfrak{x}_{\mathfrak{r}_{k}}^{2}\right)\right) e^{i 2 \pi \max \left(\alpha_{1}\left(\jmath_{j}, x_{\mathfrak{r}_{k}}^{1}\right), \alpha_{2}\left(z_{j}, x_{\mathfrak{r}_{k}}^{2}\right)\right)}\right)$, $\sigma=\max \left(\phi_{1}\left(\jmath_{j}, \mathfrak{x}_{\mathfrak{x}_{k}}^{1}\right), \phi_{2}\left(\jmath_{j}, \mathfrak{x}_{\mathfrak{r}_{k}}^{2}\right)\right)=$ $\left(\min \left(\eta_{1}\left(\jmath_{j}, x_{\mathfrak{x}_{k}}^{1}\right), \eta_{2}\left(\jmath_{j}, x_{\mathfrak{r}_{k}}^{2}\right)\right) e^{i 2 \pi \min \left(\beta_{1}\left(\jmath_{j}, x_{\mathfrak{r}_{k}}^{1}\right), \beta_{2}\left(z_{j}, x_{\mathfrak{x}_{k}}^{2}\right)\right)}\right), \quad$ and $\quad \tau=\min \left(\psi_{1}\left(\jmath_{j}, x_{\mathfrak{x}_{k}}^{1}\right), \psi_{2}\left(\jmath_{j}, \mathfrak{x}_{\mathfrak{r}_{k}}^{2}\right)\right)=$ $\left(\min \left(v_{1}\left(\jmath_{j}, x_{\mathfrak{r}_{k}}^{1}\right), v_{2}\left(\jmath_{j}, \mathfrak{x}_{\mathfrak{r}_{k}}^{2}\right)\right) e^{i 2 \pi \min \left(\gamma_{1}\left(\jmath_{j}, x_{\mathfrak{r}_{k}}^{1}\right), \gamma_{2}\left(\mathfrak{z}_{j}, x_{\mathfrak{r}_{k}}^{2}\right)\right)}\right)$ if $\left(\left(\jmath_{j}, \mathfrak{x}_{\mathfrak{r}_{k}}^{1}\right), \phi_{1}\left(\jmath_{j}, \mathfrak{x}_{\mathfrak{r}_{k}}^{1}\right), \varphi_{1}\left(\jmath_{j}, \mathfrak{x}_{\mathfrak{r}_{k}}^{1}\right), \psi_{1}\left(\jmath_{j}, \mathfrak{x}_{\mathfrak{r}_{k}}^{1}\right)\right) \in \Psi_{1}\left(\mathfrak{r}_{k}\right)$ and $\left(\left(\jmath_{j}, x_{\mathfrak{r}_{k}}^{2}\right), \phi_{2}\left(\jmath_{j}, \mathfrak{x}_{\mathfrak{r}_{k}}^{2}\right), \varphi_{2}\left(\jmath_{j}, x_{\mathfrak{r}_{k}}^{2}\right), \psi_{2}\left(\jmath_{j}, x_{\mathfrak{r}_{k}}^{2}\right)\right) \in \Psi_{2}\left(\mathfrak{r}_{k}\right)$.

Example 7: Suppose $Z=\left\{\mathfrak{z}_{1}, \jmath_{2}, \jmath_{3}\right\}$ is a universal set and $\mathfrak{R}_{1}, \mathfrak{R}_{2} \subseteq \Re_{\text {, where }} \mathfrak{R}_{1}=\left\{\mathfrak{r}_{1}, \mathfrak{r}_{2}, \mathfrak{r}_{3}\right\}$, and $\mathfrak{R}_{2}=$ $\left\{\mathfrak{r}_{1}, \mathfrak{r}_{2}, \mathfrak{r}_{4}\right\}$. Suppose $\left(\Psi_{1}, \Gamma_{1}=\left(\Delta_{1}, \Re_{1}, 5\right)\right)$ and $\left(\Psi_{2}, \Gamma_{2}=\left(\Delta_{2}, \Re_{2}, 4\right)\right)$ are CPF5-SS and CPF4-SS over $Z$ respectively. The tabular representation of CPF5-SS $\left(\Psi_{1}, \Gamma_{1}=\left(\Delta_{1}, \Re_{1}, 5\right)\right)$ is demonstrated in table 8 and tabular representation of CPF4-SS $\left(\Psi_{2}, \Gamma_{2}=\left(\Delta_{2}, \mathfrak{R}_{2}, 4\right)\right)$ is demonstrated in table 9 . Then their restricted union is demonstrated in table 11.

Table 11. The restricted union of CPF5-SS and CPF4-SS of example 7.

\begin{tabular}{|c|c|c|}
\hline$\left(\mho, \boldsymbol{\Gamma}_{1} \cup_{\mathcal{R}} \boldsymbol{\Gamma}_{2}, \mathbf{5}\right)$ & $\mathfrak{r}_{\mathbf{1}}$ & $\mathfrak{r}_{2}$ \\
\hline $\mathbf{z}_{1}$ & $2,\left(\begin{array}{c}0.5 e^{i 2 \pi(0.5)}, \\
0.1 e^{i 2 \pi(0.1)}, \\
0.2 e^{i 2 \pi(0.1)}\end{array}\right)$ & $0,\left(\begin{array}{c}0.15 e^{i 2 \pi(0.2)} \\
0.5 e^{i 2 \pi(0.4)}, \\
0.2 e^{i 2 \pi(0.2)}\end{array}\right)$ \\
\hline 32 & $3,\left(\begin{array}{c}0.69 e^{i 2 \pi(0.7)}, \\
0.1 e^{i 2 \pi(0.1)}, \\
0.1 e^{i 2 \pi(0.05)}\end{array}\right)$ & $1,\left(\begin{array}{c}0.32 e^{i 2 \pi(0.26)}, \\
0.14 e^{i 2 \pi(0.09)}, \\
0.4 e^{i 2 \pi(0.35)}\end{array}\right)$ \\
\hline 33 & $2,\left(\begin{array}{c}0.8 e^{i 2 \pi(0.8)}, \\
0.1 e^{i 2 \pi(0.1)}, \\
0.05 e^{i 2 \pi(0.05)}\end{array}\right)$ & $4,\left(\begin{array}{c}0.9 e^{i 2 \pi(0.85)}, \\
0.04 e^{i 2 \pi(0.08)}, \\
0.02 e^{i 2 \pi(0.03)}\end{array}\right)$ \\
\hline
\end{tabular}

Definition 27: Suppose $\left(\Psi_{1}, \Gamma_{1}=\left(\Delta_{1}, \Re_{1}, N_{1}\right)\right)$ and $\left(\Psi_{2}, \Gamma_{2}=\left(\Delta_{2}, \Re_{2}, N_{2}\right)\right)$ are two CPFN-SS over $Z$, then their extended intersection is signified by $\left(\Psi_{1}, \Gamma_{1}=\left(\Delta_{1}, \Re_{1}, N_{1}\right)\right) \cap_{\mathcal{E}}\left(\Psi_{2}, \Gamma_{2}=\left(\Delta_{2}, \Re_{2}, N_{2}\right)\right)$ and is define as $\left(\Omega, \Gamma_{1} \cap_{\mathcal{E}} \Gamma_{2}\right)$, where $\Gamma_{1} \cap_{\mathcal{E}} \Gamma_{2}=\left(\partial, \Re_{1} \cap \Re_{2}, \max \left(N_{1}, N_{2}\right)\right)$ and 


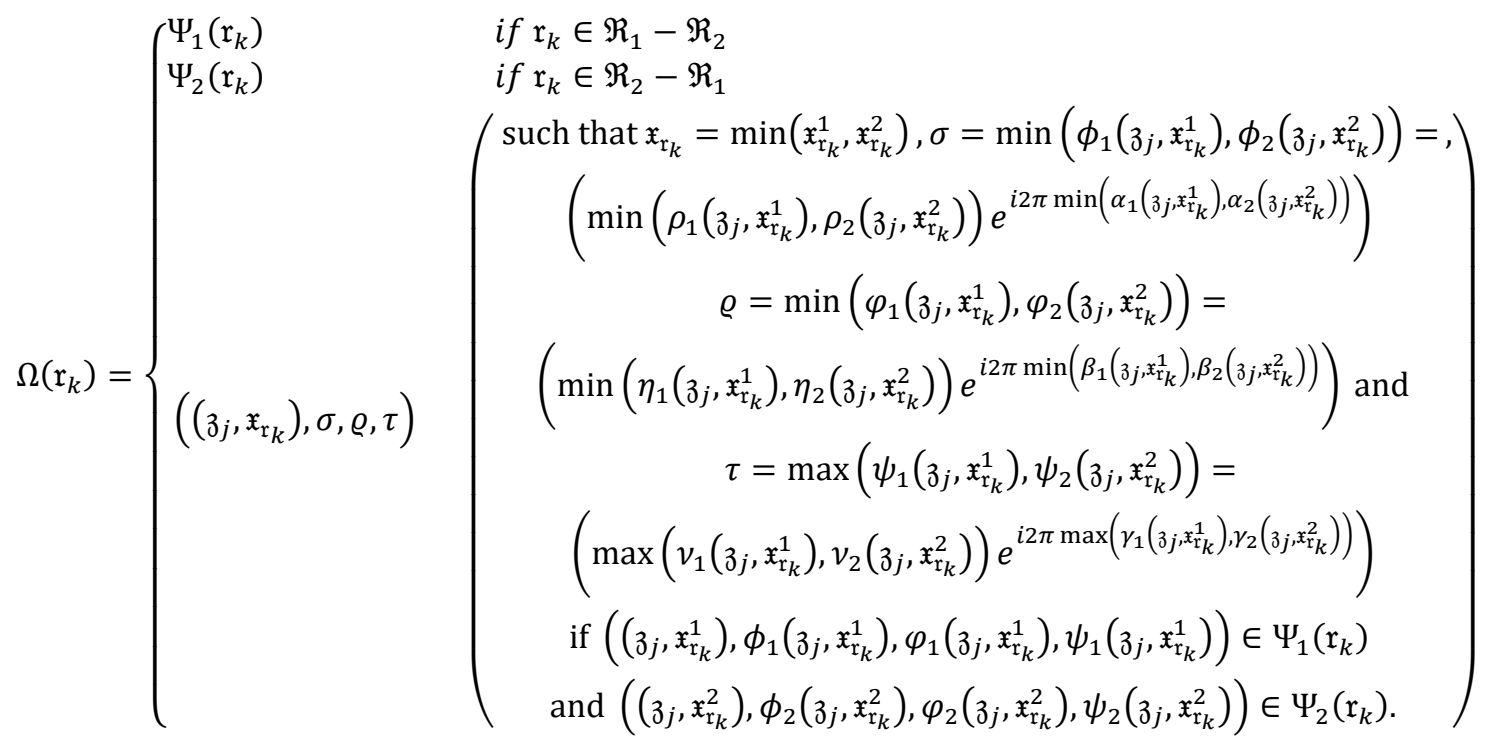

Example 8: Suppose $Z=\left\{\mathfrak{\jmath}_{1}, \jmath_{2}, \jmath_{3}\right\}$ is a universal set and $\mathfrak{R}_{1}, \Re_{2} \subseteq ß$, where $\mathfrak{R}_{1}=\left\{\mathfrak{r}_{1}, \mathfrak{r}_{2}, \mathfrak{r}_{3}\right\}$, and $\Re_{2}=$ $\left\{\mathfrak{r}_{1}, \mathfrak{r}_{2}, \mathfrak{r}_{4}\right\}$. Suppose $\left(\Psi_{1}, \Gamma_{1}=\left(\Delta_{1}, \Re_{1}, 5\right)\right)$ and $\left(\Psi_{2}, \Gamma_{2}=\left(\Delta_{2}, \Re_{2}, 4\right)\right)$ are CPF5-SS and CPF4-SS over $Z$ respectively. The tabular representation of CPF5-SS $\left(\Psi_{1}, \Gamma_{1}=\left(\Delta_{1}, \Re_{1}, 5\right)\right)$ is demonstrated in table 8 and tabular representation of CPF4-SS $\left(\Psi_{2}, \Gamma_{2}=\left(\Delta_{2}, \Re_{2}, 4\right)\right)$ is demonstrated in table 9 . Then their extended intersection is demonstrated in table 12.

Table 12. The extended intersection of CPF5-SS and CPF4-SS of example 8.

\begin{tabular}{|c|c|c|c|c|c|c|c|c|}
\hline$\left(\boldsymbol{\Omega}, \boldsymbol{\Gamma}_{1} \cap_{\mathcal{E}} \boldsymbol{\Gamma}_{2}, \mathbf{5}\right)$ & & $\mathfrak{r}_{1}$ & & $\mathfrak{r}_{2}$ & & $\mathfrak{r}_{3}$ & & $\mathfrak{r}_{4}$ \\
\hline \multirow[t]{2}{*}{$\mathbf{z}_{1}$} & \multirow[b]{2}{*}{1,} & $\left(0.4 e^{i 2 \pi(0.4)}\right.$, & & $0.05 e^{i 2 \pi(0.18)}$ & & $0.84 e^{i 2 \pi(0.8)}$ & & $0.74 e^{i 2 \pi(0.6)}$, \\
\hline & & $0.1 e^{i 2 \pi(0.1)}$ & 0 & $0.5 e^{i 2 \pi(0.4)}$ & 4, & $0.06 e^{i 2 \pi(0.07)}$ & 2, & $0.11 e^{i 2 \pi(0.2)}$ \\
\hline \multirow{3}{*}{32} & \multirow{3}{*}{2,} & $\frac{\left(0.3 e^{l 2 \pi(0.15)}\right)}{0.65 e^{i 2 \pi(0.5)}}$ & & $\frac{0.27 e^{i 2 \pi(0.3)}}{0.2 \rho^{i 2 \pi(0.15)}}$ & & $\left.0.09 e^{i 2 \pi(0.12)}\right)$ & & $\frac{\left.0.13 e^{i 2 \pi(0.1)}\right)}{\left(0.5 e^{i 2 \pi(0.47)}\right)}$ \\
\hline & & $0.1 e^{i 2 \pi(0.1)}$ & 0. & $0.14 e^{i 2 \pi(0.09)}$ & 0 & $0.4 e^{i 2 \pi(0.5)}$ & 1 & $0.2 e^{i 2 \pi(0.1)}$ \\
\hline & & $0.18 e^{i 2 \pi(0.15)}$ & ( & $0.5 e^{i 2 \pi(0.6)}$ & 0,1 & $0.3 e^{i 2 \pi(0.3)}$ & 1, & $0.2 e^{i 2 \pi(0.3)}$ \\
\hline \multirow[t]{3}{*}{33} & \multirow{3}{*}{ 1, } & $\left(0.3 e^{i 2 \pi(0.35)}\right.$, & & $0.9 e^{i 2 \pi(0.85)}$ & & $0.2 e^{i 2 \pi(0.5)}$, & & $0.6 e^{i 2 \pi(0.57)}$ \\
\hline & & $0.1 e^{i 2 \pi(0.1)}$ & 3, & $0.04 e^{i 2 \pi(0.08)}$, & 1, & $0.6 e^{i 2 \pi(0.3)}$ & 2, & $0.3 e^{i 2 \pi(0.2)}$ \\
\hline & & $0.2 e^{i 2 \pi(0.3)}$ & & $0.02 e^{i 2 \pi(0.03)}$ & & $0.19 e^{i 2 \pi(0.16)}$ & & $0.09 e^{i 2 \pi(0.1)}$ \\
\hline
\end{tabular}

Definition 28: Suppose $\left(\Psi_{1}, \Gamma_{1}=\left(\Delta_{1}, \Re_{1}, N_{1}\right)\right)$ and $\left(\Psi_{2}, \Gamma_{2}=\left(\Delta_{2}, \Re_{2}, N_{2}\right)\right)$ are two CPFN-SS over $Z$, then their extended union is signified by $\left(\Psi_{1}, \Gamma_{1}=\left(\Delta_{1}, \Re_{1}, N_{1}\right)\right) \cup_{\mathcal{E}}\left(\Psi_{2}, \Gamma_{2}=\left(\Delta_{2}, \Re_{2}, N_{2}\right)\right)$ and is define as $\left(\mho, \Gamma_{1} \cup_{\mathcal{E}} \Gamma_{2}\right)$, where $\Gamma_{1} \cup_{\mathcal{E}} \Gamma_{2}=\left(\mathcal{E}, \Re_{1} \cap \Re_{2}, \max \left(N_{1}, N_{2}\right)\right)$ and 


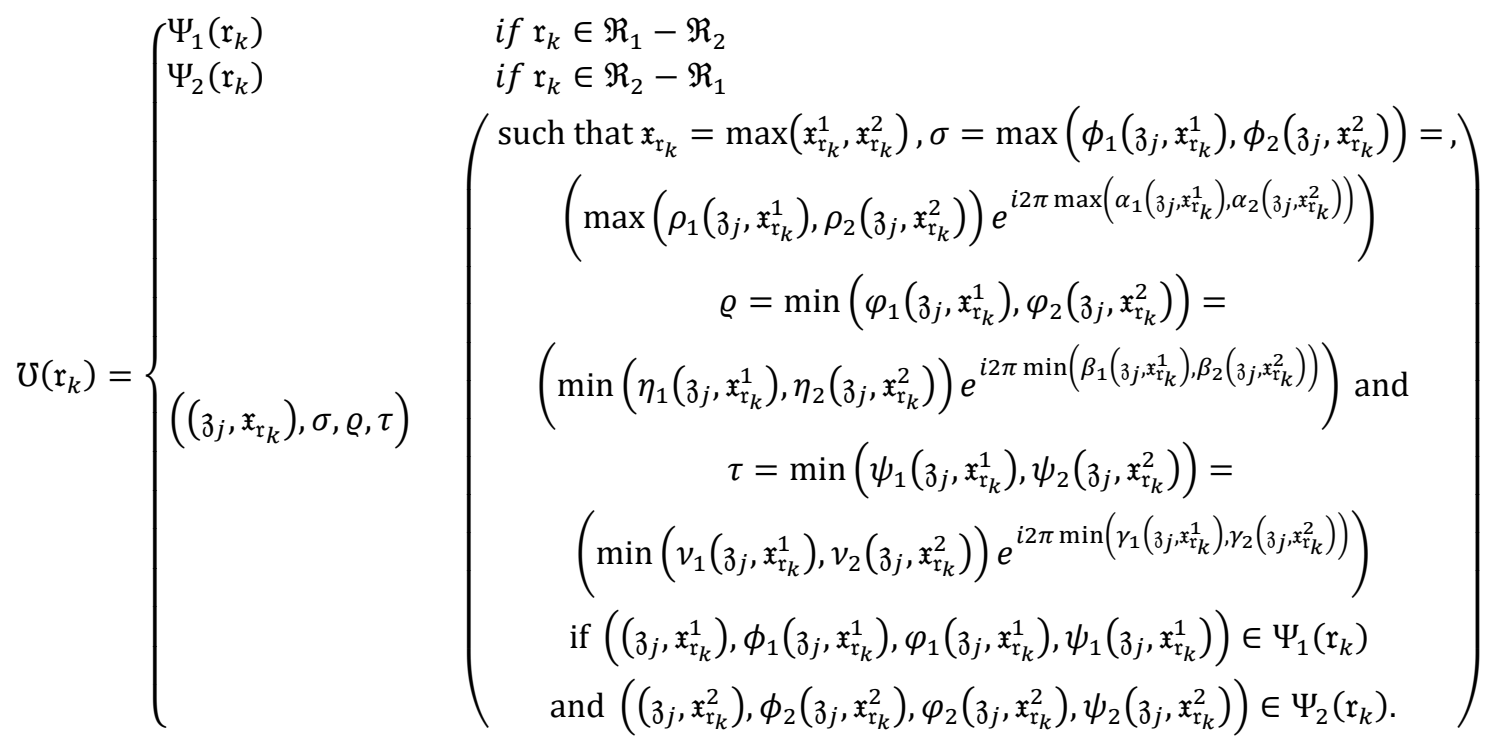

Example 9: Suppose $Z=\left\{\mathfrak{\jmath}_{1}, \jmath_{2}, \jmath_{3}\right\}$ is a universal set and $\mathfrak{R}_{1}, \Re_{2} \subseteq ß$, where $\mathfrak{R}_{1}=\left\{\mathfrak{r}_{1}, \mathfrak{r}_{2}, \mathfrak{r}_{3}\right\}$, and $\Re_{2}=$ $\left\{\mathfrak{r}_{1}, \mathfrak{r}_{2}, \mathfrak{r}_{4}\right\}$. Suppose $\left(\Psi_{1}, \Gamma_{1}=\left(\Delta_{1}, \Re_{1}, 5\right)\right)$ and $\left(\Psi_{2}, \Gamma_{2}=\left(\Delta_{2}, \Re_{2}, 4\right)\right)$ are CPF5-SS and CPF4-SS over $Z$ respectively. The tabular representation of CPF5-SS $\left(\Psi_{1}, \Gamma_{1}=\left(\Delta_{1}, \Re_{1}, 5\right)\right)$ is demonstrated in table 8 and tabular representation of CPF4-SS $\left(\Psi_{2}, \Gamma_{2}=\left(\Delta_{2}, \Re_{2}, 4\right)\right)$ is demonstrated in table 9 . Then their extended union is demonstrated in table 13.

Table 13. The extended union of CPF5-SS and CPF4-SS of example 9.

\begin{tabular}{|c|r|r|r|c|}
\hline$\left(\mho, \boldsymbol{\Gamma}_{1} \cup_{\mathcal{E}} \boldsymbol{\Gamma}_{2}, \mathbf{5}\right)$ & $\mathrm{r}_{1}$ & $\mathfrak{r}_{2}$ & $\mathfrak{r}_{3}$ & $\mathfrak{r}_{4}$ \\
\hline $3_{1}$ & $2,\left(\begin{array}{c}0.5 e^{i 2 \pi(0.5)}, \\
0.1 e^{i 2 \pi(0.1)}, \\
0.2 e^{i 2 \pi(0.1)}\end{array}\right)$ & $0,\left(\begin{array}{c}0.15 e^{i 2 \pi(0.2)}, \\
0.5 e^{i 2 \pi(0.4)}, \\
0.2 e^{i 2 \pi(0.2)}\end{array}\right)$ & $4,\left(\begin{array}{c}0.84 e^{i 2 \pi(0.8)}, \\
0.06 e^{i 2 \pi(0.07)} \\
0.09 e^{i 2 \pi(0.12)}\end{array}\right)$ & $2,\left(\begin{array}{c}0.74 e^{i 2 \pi(0.6)}, \\
0.11 e^{i 2 \pi(0.2)} \\
0.13 e^{i 2 \pi(0.1)}\end{array}\right)$ \\
\hline 32 & $3,\left(\begin{array}{c}0.69 e^{i 2 \pi(0.7)}, \\
0.1 e^{i 2 \pi(0.1)}, \\
0.1 e^{i 2 \pi(0.05)}\end{array}\right)$ & $1,\left(\begin{array}{c}0.32 e^{i 2 \pi(0.26)}, \\
0.14 e^{i 2 \pi(0.09)}, \\
0.4 e^{i 2 \pi(0.35)}\end{array}\right)$ & $0,\left(\begin{array}{c}0.15 e^{i 2 \pi(0.17)}, \\
0.4 e^{i 2 \pi(0.5)}, \\
0.3 e^{i 2 \pi(0.3)}\end{array}\right)$ & $1,\left(\begin{array}{c}0.5 e^{i 2 \pi(0.47)}, \\
0.2 e^{i 2 \pi(0.1)}, \\
0.2 e^{i 2 \pi(0.3)}\end{array}\right)$ \\
\hline 33 & $2,\left(\begin{array}{c}0.8 e^{i 2 \pi(0.8)}, \\
0.1 e^{i 2 \pi(0.1)}, \\
0.05 e^{i 2 \pi(0.05)}\end{array}\right)$ & $4,\left(\begin{array}{c}0.9 e^{i 2 \pi(0.85)}, \\
0.04 e^{i 2 \pi(0.08)}, \\
0.02 e^{i 2 \pi(0.03)}\end{array}\right)$ & $1,\left(\begin{array}{c}0.2 e^{i 2 \pi(0.5)}, \\
0.6 e^{i 2 \pi(0.3)}, \\
0.19 e^{i 2 \pi(0.16)}\end{array}\right)$ & $2,\left(\begin{array}{c}0.6 e^{i 2 \pi(0.57)}, \\
0.3 e^{i 2 \pi(0.2)}, \\
0.09 e^{i 2 \pi(0.1)}\end{array}\right)$ \\
\hline
\end{tabular}

\section{Applications of the CPFN-SS}

In this section, we define the algorithm for the real-world issues that are existing in the environment of CPFN-SS. We give two real-life examples (Performance assessment of e-waste recycling programs and Predication about champions of FIFA world cup 2022 through audience poll) of DM in the environment of CPFN-SS to show the functionality and practicality of our established work.

\subsection{Algorithm for the data in the form CPFN-SS}

For the selection of alternative in the environment of CPFN-SS, we initiated the following algorithm.

1. Utilize $Z=\left\{z_{1}, z_{2}, z_{3}, \ldots, z_{n}\right\}$ as universal set

2. Utilize $\Re=\left\{\mathfrak{r}_{1}, \mathfrak{r}_{2}, \mathfrak{r}_{3}, \ldots, \mathfrak{r}_{m}\right\} \subseteq ß$ as a set of attributes.

3. Make a CPFN-SS in tabular form . 
4. Make the tables of complex positive pole $(\mathrm{CPP}) \phi$, complex neutral pole $(\mathrm{CnP}) \varphi$, and complex negative pole (CNP) $\psi$.

5. Compute the comparison tables for $\mathrm{CPP}, \mathrm{CnP}$, and CNP. (since the $\mathrm{CPP}, \mathrm{CnP}$, and $\mathrm{CNP}$ are in the form of CFS so for the comparison of the two CFNs we will use lexicographical order for example, if $\phi_{1}=\rho_{1} e^{i 2 \pi\left(\alpha_{1}\right)}, \phi_{2}=\rho_{2} e^{i 2 \pi\left(\alpha_{2}\right)}$ are two PP. If $\rho_{1}<\rho_{2}$ then we say that $\phi_{1}<\phi_{2}$. But if $\rho_{1}=\rho_{2}$ then we see the imaginary part i.e. if $\alpha_{1}<\alpha_{2}$ then $\phi_{1}<\phi_{2}$. If both $\rho_{1}=\rho_{2}$ and $\alpha_{1}=\alpha_{2}$ then we have that $\phi_{1}=\phi_{2}$.

6. Make the score tables for $\mathrm{CPP}, \mathrm{CnP}$, and CNP.

7. Adding the scores of $\mathrm{CnP}$ and CNP.

8. Evaluate the final score by subtracting the sum of scores of $\mathrm{CnP}$ and CNP from the score of PP.

9. Consider the highest score with greatest grades, if it is in $k-t h$ row, then we will select option $z_{k}$, $1 \leq k \leq n$.

\subsection{Performance assessment of e-waste recycling programs}

E-waste is short for electronic waste, .i.e. waste generated from surplus, broken, and obsolete electronic gadgets. People also called it e-scraps. Ordinarily, these electronics frequently have harmful chemicals and risky materials. And if we don't dispose of these electronics accurately, it can cause the discharge of harmful materials into our natural environment. E-waste recycling then suggests the reprocessing of these e-wastes. It is a procedure to recover material from e-waste. Thusly, we can utilize them in new electronic items. Today e-waste recycling is one of the most debated problems in the world because of its capabilities to decrease environmental perils and contamination. In the following example, we will use the CPFN-SS to evaluate the performance of e-waste recycling programs (e-WRPs).

Example 10: Suppose an electronics company needs the most appropriate e-WRP which decreases the harmful environmental influence of e-waste, and simultaneously, developing its business. For selection and assessment of the e-WRP the company hire a decision-maker. The decision-maker found various potential e-WRPs and assessment criteria through a complete analysis. Four alternative e-WRPs include

- $\boldsymbol{z}_{\mathbf{1}}=$ Retrieval of plastic and metals from e-waste

- $\quad \mathbf{z}_{2}=$ Retrieval of glass and other recycling substances from e-waste

- $\quad z_{3}=$ Retrieval of mercury and valuable plastic

- $\quad \mathbf{z}_{4}=$ Retrieval of circuit boards and other valuable substances

Three most significant criteria are found for assessing the performance of appropriate e-WRP including

- $\mathfrak{r}_{1}=$ Social criteria

- $\mathfrak{r}_{2}=$ Technical criteria

- $\mathfrak{r}_{3}=$ Environmental criteria 
Level 1

E-Waste Recycling Program Performance Evaluation

Level 2

Criteria

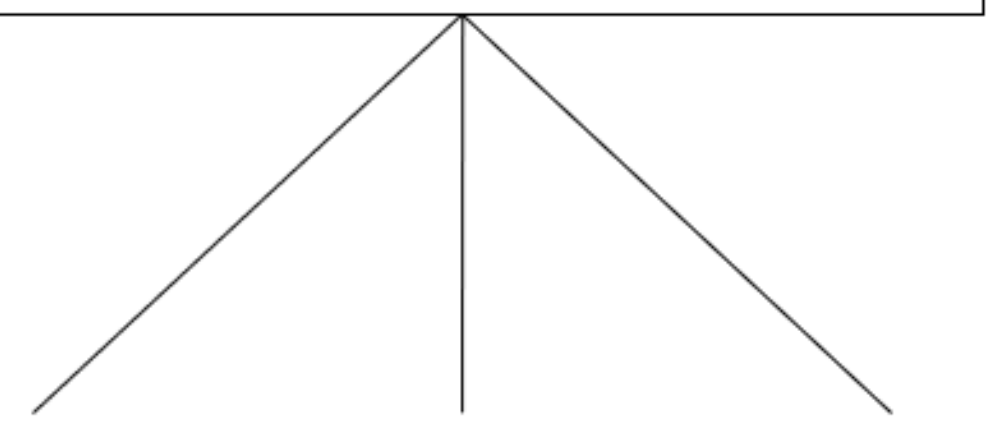

Level 3

Alternatives

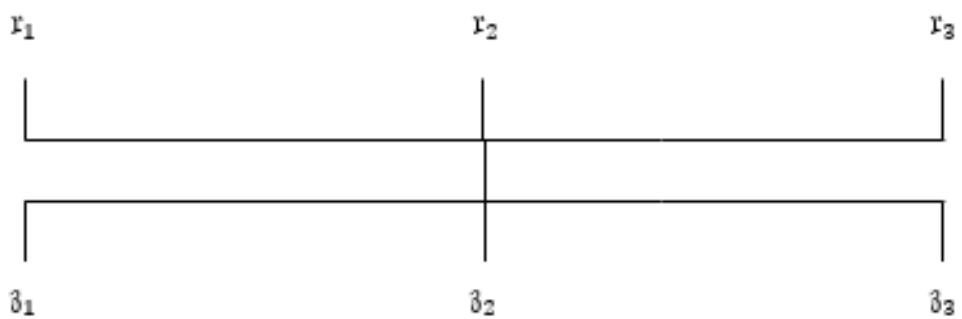

Legend

$\mathrm{r}_{1}$ : Social criteria

$\mathrm{r}_{2}=$ Technical criteria $\quad \mathrm{r}_{3}=$ Environmental criteria

$3_{i}(i=1,2, \ldots, n)$

E-Waste Recycling Program

Figure 1. The hierarchical structure of e-WRP performance assessment.

The e-WRP performance assessment producer starts with directing the decision maker to give grades in the shape of circle stars and circle hole to the alternatives on the basis of criteria which is display in table 14.

Table 14. The information given by decision- maker.

\begin{tabular}{|c|c|c|c|}
\hline $\boldsymbol{Z}$ & $\mathfrak{r}_{1}$ & $\mathbf{r}_{2}$ & $\mathbf{r}_{3}$ \\
\hline $\mathbf{3}_{1}$ & $\circledast \circledast$ & $\star \circledast$ & 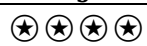 \\
\hline $3_{2}$ & $\circledast \circledast \circledast$ & $\star$ & $\circledast$ \\
\hline 33 & $\star$ & $\star \circledast \circledast \circledast$ & ○) \\
\hline $3_{4}$ & $\circledast$ & () & 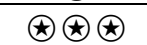 \\
\hline
\end{tabular}

We can link the set $\mathfrak{X}=\{0,1,2,3,4\}$ with the circle stars and circle hole displayed in table 14 by saying that 4 characterizes $" \circledast \circledast \circledast \circledast$ ", 3 characterizes $" \circledast \circledast \circledast$ ", 2 characterizes $" \circledast \circledast$ ", 1 signifies $" \circledast "$, and 0 characterizes " $\odot "$ ". Then the 5-SS $(\Delta, \Re, 5)$ is defined as

$$
\begin{aligned}
& \Delta\left(\mathfrak{r}_{1}\right)=\left\{\left(\jmath_{1}, 2\right),\left(z_{2}, 3\right),\left(\jmath_{3}, 1\right),\left(z_{4}, 1\right)\right\} \\
& \Delta\left(\mathfrak{r}_{2}\right)=\left\{\left(3_{1}, 2\right),\left(z_{2}, 1\right),\left(\jmath_{3}, 4\right),\left(3_{4}, 0\right)\right\}
\end{aligned}
$$




$$
\Delta\left(\mathfrak{r}_{3}\right)=\left\{\left(3_{1}, 4\right),\left(3_{2}, 1\right),\left(3_{3}, 0\right),\left(3_{4}, 3\right)\right\}
$$

In table 15, we display the tabular form of 5-SS

Table 15. 5-SS in tabular form.

\begin{tabular}{|c|c|c|c|}
\hline$(\boldsymbol{\Delta}, \boldsymbol{R}, \mathbf{5})$ & $\mathfrak{r}_{\mathbf{1}}$ & $\mathfrak{r}_{\mathbf{2}}$ & $\mathfrak{r}_{\mathbf{3}}$ \\
\hline $\boldsymbol{3}_{1}$ & 2 & 2 & 4 \\
\hline $\boldsymbol{3}_{2}$ & 3 & 1 & 1 \\
\hline $\boldsymbol{3}_{3}$ & 1 & 4 & 0 \\
\hline $\mathbf{3}_{4}$ & 1 & 0 & 3 \\
\hline
\end{tabular}

$\phi, \varphi$, and $\psi$ will satisfy the following grading criteria. If grade is 0 , then $0.0 \leq \phi(3)<0.2$, (i.e. $0.0 \leq$ $\left.\frac{\rho(3)+\alpha(3)}{2} \leq 0.2\right)$ and $0 \leq \phi(3)+\varphi(3)+\psi(3) \leq 1$, i.e. $0 \leq \rho(3)+\eta(3)+v(3) \leq 1$ and $0 \leq \alpha(3)+\beta(3)+\gamma(3) \leq$ 1 , where $0 \leq \rho(3), \eta(3), v(3), \alpha(3), \beta(3), \gamma(z) \leq 1$ for each $z \in Z$. In the similar manner, if grade is 1 then $0.2 \leq$ $\phi(3)<0.4$, if grade is 2 then $0.4 \leq \phi(3)<0.6$, if grade is 3 then $0.6 \leq \phi(3)<0.8$, and if grade is 4 then $0.8 \leq$ $\phi(\jmath) \leq 1.0$. Then the CPF5-SS is established below:

$$
\begin{aligned}
& \Psi\left(\mathfrak{r}_{1}\right)=\left\{\begin{array}{c}
\left(\left(3_{1}, 2\right),\left(\begin{array}{c}
0.4 e^{i 2 \pi(0.5)}, 0.2 e^{i 2 \pi(0.1)}, \\
0.3 e^{i 2 \pi(0.15)}
\end{array}\right),\left(\left({ }_{2}, 3\right),\left(\begin{array}{c}
0.65 e^{i 2 \pi(0.7)}, 0.1 e^{i 2 \pi(0.1)}, \\
0.1 e^{i 2 \pi(0.05)}
\end{array}\right)\right)\right. \\
\left(\left(\left(3_{3}, 1\right),\left(\begin{array}{c}
0.3 e^{i 2 \pi(0.35)}, 0.4 e^{i 2 \pi(0.2)} \\
0.2 e^{i 2 \pi(0.3)}
\end{array}\right),\left(\left({ }_{3}, 1\right),\left(\begin{array}{c}
0.35 e^{i 2 \pi(0.45)}, 0.3 e^{i 2 \pi(0.3)}, \\
0.25 e^{i 2 \pi(0.1)}
\end{array}\right)\right)\right.\right.
\end{array}\right\}
\end{aligned}
$$

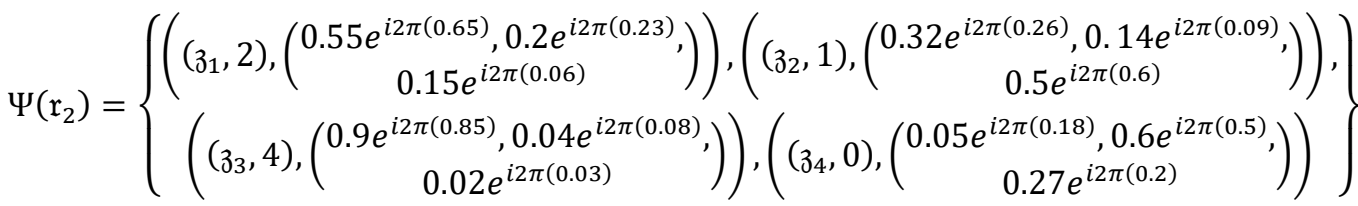

$$
\begin{aligned}
& \Psi\left(\mathfrak{r}_{3}\right)=\left\{\begin{array}{l}
\left(\left({ }_{1}, 4\right),\left(\begin{array}{c}
0.84 e^{i 2 \pi(0.8)}, 0.06 e^{i 2 \pi(0.07)}, \\
\left.0.09 e^{i 2 \pi(0.12)}\right)
\end{array}\right),\left(\left({ }_{2}, 1\right),\left(\begin{array}{c}
0.2 e^{i 2 \pi(0.5)}, 0.6 e^{i 2 \pi(0.3)}, \\
0.19 e^{i 2 \pi(0.16)}
\end{array}\right)\right),\right. \\
\left(\left({ }_{3}, 0\right),\left(\begin{array}{c}
0.15 e^{i 2 \pi(0.17)}, 0.4 e^{i 2 \pi(0.5)} \\
0.3 e^{i 2 \pi(0.3)}
\end{array}\right)\right),\left(\left(3_{4}, 3\right),\left(\begin{array}{c}
0.75 e^{i 2 \pi(0.65)}, 0.1 e^{i 2 \pi(0.1)} \\
0.1 e^{i 2 \pi(0.2)}
\end{array}\right)\right)
\end{array}\right\}
\end{aligned}
$$

In table 16, we display the tabular form of CPF5-SS.

Table 16. The Tabular representation of CPF5-SS of example 10.

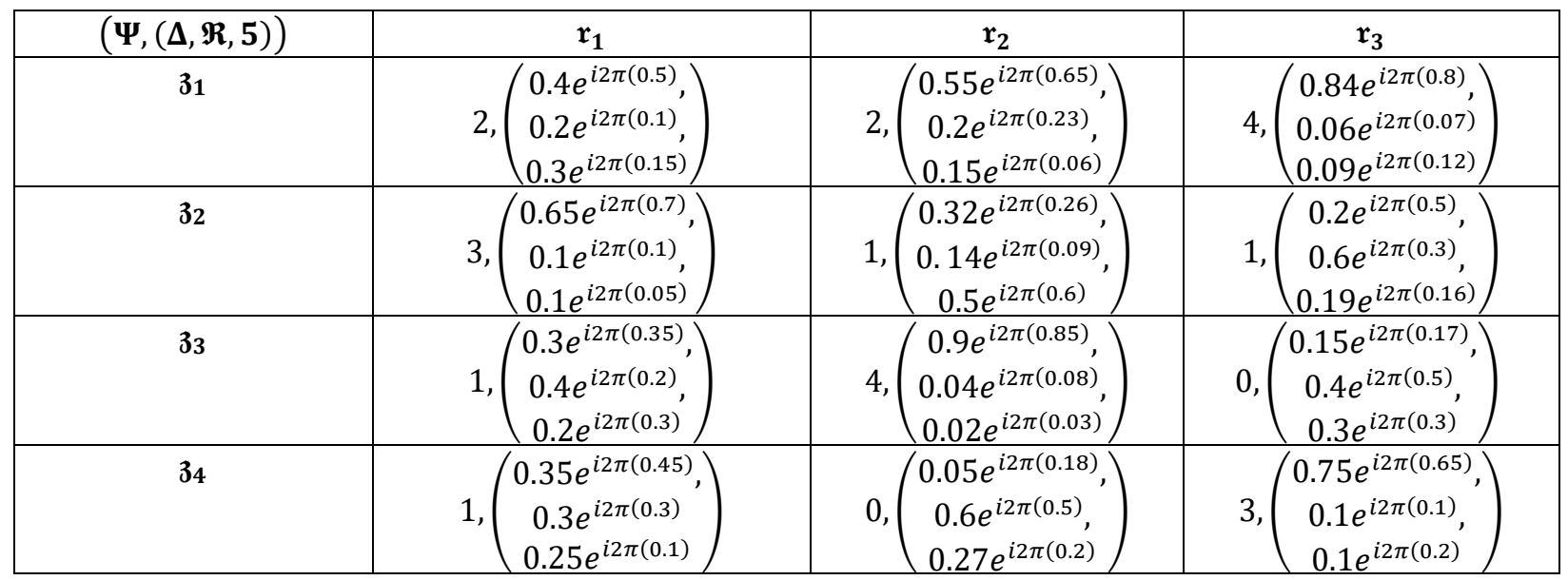


The tabular representation of CPP is demonstrated in table 17. We make the comparison table for CPP which is demonstrated in table 18. Afterwards, we determine the CPP score for each e-WRP with the sum of grades $\left(\sum_{i=1}^{3} \mathfrak{x}_{\mathfrak{r}_{i}}\right)$ by subtracting the column sum from the row sum of table 18. and demonstrated in table 19.

Table 17. The tabular representation of CPP of example 10.

\begin{tabular}{|c|c|c|c|}
\hline $\boldsymbol{\phi}$ & $\mathfrak{r}_{1}$ & $\mathfrak{r}_{2}$ & $\mathfrak{r}_{3}$ \\
\hline $\mathbf{z}_{1}$ & $0.4 e^{i 2 \pi(0.5)}$ & $0.55 e^{i 2 \pi(0.65)}$ & $0.84 e^{i 2 \pi(0.8)}$ \\
\hline $\mathbf{z}_{2}$ & $0.65 e^{i 2 \pi(0.7)}$ & $0.32 e^{i 2 \pi(0.26)}$ & $0.2 e^{i 2 \pi(0.5)}$ \\
\hline $\mathbf{3}_{3}$ & $0.3 e^{i 2 \pi(0.35)}$ & $0.9 e^{i 2 \pi(0.85)}$ & $0.15 e^{i 2 \pi(0.17)}$ \\
\hline $\mathbf{3}_{4}$ & $0.35 e^{i 2 \pi(0.45)}$ & $0.05 e^{i 2 \pi(0.18)}$ & $0.75 e^{i 2 \pi(0.65)}$ \\
\hline
\end{tabular}

Table 18. The comparison table of CPP of example 10.

\begin{tabular}{|c|c|c|c|c|}
\hline. & $3_{1}$ & $3_{2}$ & $3_{3}$ & $3_{4}$ \\
\hline $\boldsymbol{z}_{1}$ & 3 & 2 & 2 & 3 \\
\hline $\mathbf{3}_{2}$ & 1 & 3 & 2 & 2 \\
\hline $\mathbf{3}_{3}$ & 1 & 1 & 3 & 1 \\
\hline $\mathbf{3}_{4}$ & 0 & 1 & 2 & 3 \\
\hline
\end{tabular}

Table 19. The CPP score of e-WRP.

\begin{tabular}{|c|c|c|c|c|}
\hline & $\begin{array}{c}\text { Grade sum } \\
\left(\sum_{i=1}^{\mathbf{3}} \mathfrak{x}_{\mathbf{r}_{i}}\right)\end{array}$ & $\begin{array}{c}\text { Row sum } \\
\left(\mathcal{R} \boldsymbol{S}_{\mathbf{1}}\right)\end{array}$ & $\begin{array}{c}\text { Column sum } \\
\left(\boldsymbol{C} \boldsymbol{S}_{\mathbf{1}}\right)\end{array}$ & $\boldsymbol{\nabla}_{\mathbf{1}}=\boldsymbol{R}_{\mathbf{1}}-\boldsymbol{C} \boldsymbol{S}_{\mathbf{1}}$ \\
\hline $\mathbf{3}_{\mathbf{1}}$ & 8 & 10 & 5 & 5 \\
\hline $\mathbf{3}_{2}$ & 6 & 8 & 7 & 1 \\
\hline $\mathbf{3}_{3}$ & 5 & 6 & 9 & -3 \\
\hline $\mathbf{3}_{4}$ & 4 & 6 & 9 & -3 \\
\hline
\end{tabular}

Now similarly the tabular representation of $\mathrm{CnP}$ is demonstrated in table 20. We make the comparison table for $\mathrm{CnP}$ which is demonstrated in table 21. Afterwards, we determine the $\mathrm{CnP}$ score for each e-WRP with the sum of grades $\left(\sum_{i=1}^{3} \mathfrak{x}_{\mathfrak{r}_{i}}\right)$ by subtracting the column sum from the row sum of table 21 and demonstrated in table 22.

Table 20. The tabular representation of CnP of example 10.

\begin{tabular}{|c|c|c|c|}
\hline $\boldsymbol{\varphi}$ & $\mathfrak{r}_{1}$ & $\mathfrak{r}_{2}$ & $\mathfrak{r}_{3}$ \\
\hline $\mathbf{z}_{1}$ & $0.2 e^{i 2 \pi(0.1)}$ & $0.2 e^{i 2 \pi(0.23)}$ & $0.06 e^{i 2 \pi(0.07)}$ \\
\hline $\mathbf{z}_{2}$ & $0.1 e^{i 2 \pi(0.1)}$ & $0.14 e^{i 2 \pi(0.09)}$ & $0.6 e^{i 2 \pi(0.3)}$ \\
\hline $\mathbf{3}_{3}$ & $0.4 e^{i 2 \pi(0.2)}$ & $0.04 e^{i 2 \pi(0.08)}$ & $0.4 e^{i 2 \pi(0.5)}$ \\
\hline $\mathbf{z}_{4}$ & $0.3 e^{i 2 \pi(0.3)}$ & $0.6 e^{i 2 \pi(0.5)}$ & $0.1 e^{i 2 \pi(0.1)}$ \\
\hline
\end{tabular}


Table 21. The comparison table of CnP of example 10.

\begin{tabular}{|c|c|c|c|c|}
\hline & $3_{1}$ & 32 & 33 & 3 \\
\hline $\mathbf{z}_{1}$ & 3 & 2 & 2 & 0 \\
\hline $\mathbf{3 2}_{3}$ & 1 & 3 & 2 & 1 \\
\hline $\mathbf{3}_{\mathbf{3}}$ & 2 & 1 & 3 & 2 \\
\hline
\end{tabular}

Table 22. The CnP score of e-WRP.

\begin{tabular}{|c|c|c|c|c|}
\hline & $\begin{array}{c}\text { Grade sum } \\
\left(\sum_{i=1}^{\mathbf{3}} \mathfrak{x}_{\mathfrak{r}_{i}}\right)\end{array}$ & $\begin{array}{c}\text { Row sum } \\
\left(\mathcal{R S}_{\mathbf{2}}\right)\end{array}$ & $\begin{array}{c}\text { Column sum } \\
\left(\boldsymbol{C S}_{\mathbf{2}}\right)\end{array}$ & $\boldsymbol{\nabla}_{\mathbf{2}}=\mathcal{R S}_{\mathbf{2}}-\boldsymbol{C S}_{\mathbf{2}}$ \\
\hline $\boldsymbol{3}_{\mathbf{1}}$ & 8 & 6 & 9 & -3 \\
\hline $\mathbf{3}_{2}$ & 6 & 7 & 8 & -1 \\
\hline $\boldsymbol{3}_{3}$ & 5 & 8 & 7 & 1 \\
\hline $\mathbf{3}_{4}$ & 4 & 9 & 6 & 3 \\
\hline
\end{tabular}

Next the tabular representation of CNP is demonstrated in table 23. We make the comparison table for CNP which is demonstrated in table 24. Afterwards, we determine the CNP score for each e-WRP with the sum of grades $\left(\sum_{i=1}^{3} \mathfrak{x}_{\mathfrak{r}_{i}}\right)$ by subtracting the column sum from the row sum of table 24 . and demonstrated in table 25.

Table 23. The tabular representation of CNP of example 10.

\begin{tabular}{|c|c|c|c|}
\hline $\boldsymbol{\psi}$ & $\mathfrak{r}_{\mathbf{1}}$ & $\mathfrak{r}_{\mathbf{2}}$ & $\mathfrak{r}_{\mathbf{3}}$ \\
\hline $\mathbf{z}_{\mathbf{1}}$ & $0.3 e^{i 2 \pi(0.15)}$ & $0.15 e^{i 2 \pi(0.06)}$ & $0.09 e^{i 2 \pi(0.12)}$ \\
\hline $\mathbf{z}_{\mathbf{2}}$ & $0.1 e^{i 2 \pi(0.05)}$ & $0.5 e^{i 2 \pi(0.6)}$ & $0.19 e^{i 2 \pi(0.16)}$ \\
\hline $\mathbf{z}_{\mathbf{3}}$ & $0.2 e^{i 2 \pi(0.3)}$ & $0.02 e^{i 2 \pi(0.03)}$ & $0.3 e^{i 2 \pi(0.3)}$ \\
\hline
\end{tabular}

Table 24. The comparison table of CNP of example 10.

\begin{tabular}{|c|c|c|c|c|}
\hline & 31 & 32 & 33 & 3 \\
\hline $\mathbf{3 1}_{\mathbf{3}}$ & 3 & 1 & 2 & 1 \\
\hline $\mathbf{3}_{\mathbf{3}}$ & 2 & 3 & 3 & 2 \\
\hline $\mathbf{3 4}$ & 1 & 1 & 2 & 1 \\
\hline
\end{tabular}

Table 25. The CNP score of each e-WRP.

\begin{tabular}{|c|c|c|c|c|}
\hline & $\begin{array}{l}\text { Grade sum } \\
\left(\sum^{3} \mathfrak{x}_{\mathfrak{r}_{i}}\right)\end{array}$ & $\begin{array}{l}\text { Row sum } \\
\qquad\left(\mathcal{R} \boldsymbol{S}_{3}\right)\end{array}$ & $\begin{array}{l}\text { Column sum } \\
\qquad\left(\mathcal{C S}_{3}\right)\end{array}$ & $\nabla_{3}=\mathcal{R} \delta_{3}-\mathcal{C} \mathcal{S}_{3}$ \\
\hline $3_{1}$ & 8 & 7 & 8 & -1 \\
\hline 32 & 6 & 8 & 6 & 2 \\
\hline $3_{3}$ & 5 & 6 & 8 & -2 \\
\hline 34 & 4 & 8 & 7 & 1 \\
\hline
\end{tabular}


At the end, add the scores of $\mathrm{CnP}$ and CNP which is displayed in table 26 and then subtract it by the score of PP as demonstrated in table 27.

Table 26. Addition of CnP and CNP scores of example 10.

\begin{tabular}{|c|c|c|c|c|}
\hline & $\begin{array}{c}\text { Grade sum } \\
\left(\sum_{i=1}^{\mathbf{3}} \mathfrak{x}_{\mathbf{r}_{i}}\right)\end{array}$ & $\boldsymbol{\nabla}_{\mathbf{2}}$ & $\boldsymbol{\nabla}_{\mathbf{3}}$ & $\boldsymbol{\nabla}_{\mathbf{4}}=\boldsymbol{\nabla}_{\mathbf{2}}+\boldsymbol{\nabla}_{\mathbf{3}}$ \\
\hline $\mathbf{z}_{\mathbf{1}}$ & 8 & -3 & -1 & -4 \\
\hline $\mathbf{3}_{2}$ & 6 & -1 & 2 & -1 \\
\hline $\mathbf{3}_{3}$ & 5 & 1 & -2 & 4 \\
\hline $\mathbf{3}_{4}$ & 4 & 3 & 1 & \\
\hline
\end{tabular}

Table 27. Table of final score with grades of example 10.

\begin{tabular}{|c|c|c|c|c|}
\hline & $\begin{array}{c}\text { Grade sum } \\
\left(\sum_{i=1}^{\mathbf{3}} \mathfrak{x}_{\mathbf{r}_{i}}\right)\end{array}$ & $\boldsymbol{\nabla}_{\mathbf{1}}$ & $\boldsymbol{\nabla}_{\mathbf{4}}$ & $\begin{array}{c}\text { Final Score } \\
=\boldsymbol{\nabla}_{\mathbf{1}}-\boldsymbol{\nabla}_{\mathbf{4}}\end{array}$ \\
\hline $\boldsymbol{3}_{\mathbf{1}}$ & 8 & 5 & -4 & 9 \\
\hline $\mathbf{z}_{2}$ & 6 & 1 & 1 & 0 \\
\hline $\mathbf{3}_{3}$ & 5 & -3 & -1 & -2 \\
\hline $\mathbf{3}_{4}$ & 4 & -3 & 4 & -7 \\
\hline
\end{tabular}

After the whole process of the assessment of the e-WRPs, the decision-maker concluded that the e-WRP $z_{1}$ has good performance as compared to other e-WRPs as it has maximum grades i.e. 8 with the highest score 9 as demonstrated in table 27. This leads the electronics company in a present situation to select the most suitable e-WRP.

\subsection{Predication about champions of FIFA world cup 2022 through audience poll}

The FIFA world cup is an international football competition between men's national teams of different countries who are associate with the Federation Internationale de Football Association (FIFA). The championship has been given every four years since the opening tournament in 1930. In this subsection, we will show through the following example that how we utilize CPFN-SS to conduct an audience poll for the next champions of FIFA world cup 2022.

Example 11: Suppose we want to conduct an audience poll to get the people's point of view that which team will win the world cup 2022. We provided top three ranked football teams i.e. $z_{1}=$ Belgium, $z_{2}=$ France, and $z_{3}=$ Brazil, and asked people to vote for these teams in the form of circle stars and circle hole based on the following attributes

- $\mathrm{r}_{1}=$ Game plan

- $\mathfrak{r}_{2}=$ Skills, speed, and shot accuracy of the players of each team

- $\mathfrak{r}_{3}=$ Performances of each team in previous world cups

which is demonstrated in the table 28. 
Table 28. The information given by audience.

\begin{tabular}{|c|c|c|c|}
\hline $\mathcal{Z}$ & $\mathfrak{r}_{1}$ & $\mathfrak{r}_{2}$ & $\mathfrak{r}_{3}$ \\
\hline $\mathbf{3}_{1}$ & $\circledast \circledast$ & $\star \star \circledast$ & $\circledast \circledast \circledast$ \\
\hline 32 & $\star$ & () & $\circledast \circledast \circledast$ \\
\hline $3_{3}$ & $\star \star \circledast$ & $\circledast \circledast$ & $\circledast \circledast \circledast$ \\
\hline
\end{tabular}

We can relate the set $\mathfrak{X}=\{0,1,2,3\}$ with the circle stars and circle hole displayed in table 28 by saying that 3 characterizes $" \circledast \circledast \circledast$ ", 2 characterizes $" \circledast \circledast$ ", 1 signifies "®", and 0 characterizes " $\odot$ ". Then the 4-SS $(\Delta, \Re, 4)$ is demonstrated as

$$
\begin{aligned}
& \Delta\left(\mathrm{r}_{1}\right)=\left\{\left(\mathrm{z}_{1}, 2\right),\left(\mathrm{z}_{2}, 3\right),\left(\mathrm{z}_{3}, 3\right)\right\} \\
& \Delta\left(\mathfrak{r}_{2}\right)=\left\{\left(3_{1}, 1\right),\left(3_{2}, 0\right),\left(\jmath_{3}, 3\right)\right\} \\
& \Delta\left(\mathfrak{r}_{3}\right)=\left\{\left(3_{1}, 3\right),\left(3_{2}, 2\right),\left(3_{3}, 3\right)\right\}
\end{aligned}
$$

In table 29, we display the tabular form of 4-SS

Table 29. 4-SS in tabular form.

\begin{tabular}{|c|c|c|c|}
\hline$(\boldsymbol{\Delta}, \mathfrak{R}, \mathbf{4})$ & $\mathfrak{r}_{\mathbf{1}}$ & $\mathfrak{r}_{\mathbf{2}}$ & $\mathfrak{r}_{\mathbf{3}}$ \\
\hline $\boldsymbol{z}_{1}$ & 2 & 3 & 3 \\
\hline $\boldsymbol{3}_{2}$ & 1 & 0 & 3 \\
\hline $\boldsymbol{3}_{3}$ & 3 & 2 & 3 \\
\hline
\end{tabular}

$\phi, \varphi$, and $\psi$ will satisfy the following grading criteria. If grade is 0 , then $0.0 \leq \phi(3)<0.25$, (i.e. $0.0 \leq$ $\left.\frac{\rho(3)+\alpha(3)}{2} \leq 0.25\right)$ and $0 \leq \phi(3)+\varphi(3)+\psi(3) \leq 1$, i.e. $0 \leq \rho(3)+\eta(3)+v(3) \leq 1$ and $0 \leq \alpha(3)+\beta(3)+\gamma(3) \leq$ 1 , where $0 \leq \rho(3), \eta(z), v(z), \alpha(3), \beta(3), \gamma(z) \leq 1$ for each $z \in Z$. In the similar manner, if grade is 1 then $0.25 \leq \phi(3)<0.5$, if grade is 2 then $0.5 \leq \phi(3)<0.75$, if grade is 3 then $0.75 \leq \phi(3) \leq 1.0$ Then the CPF4$\mathrm{SS}$ is established below:

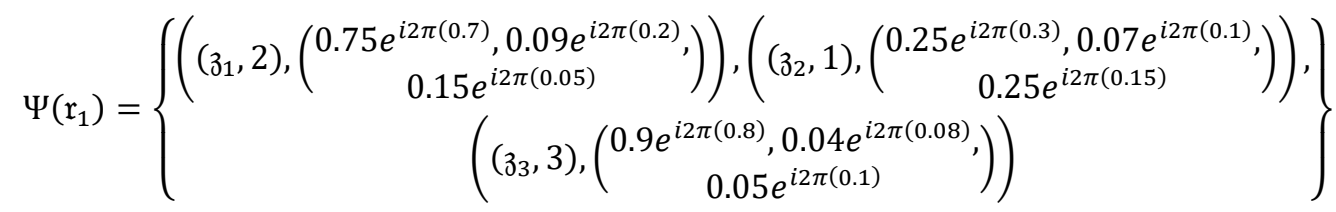

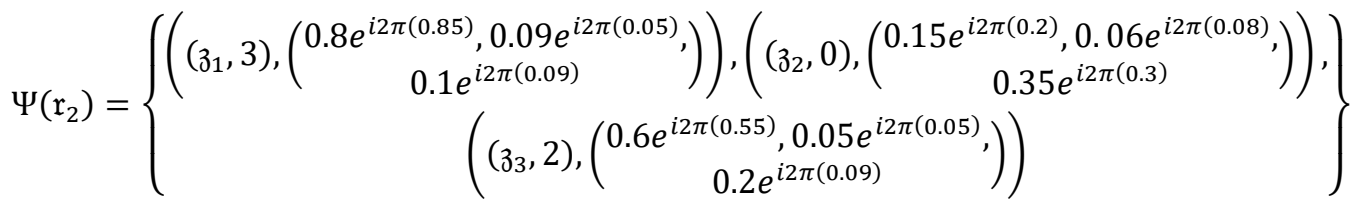

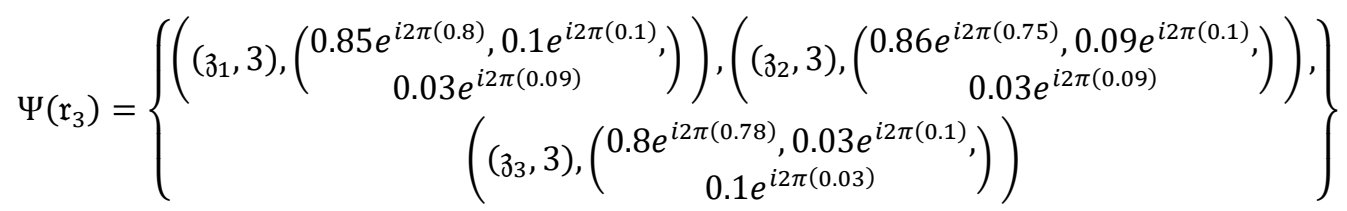

In table 30, we display the tabular form of CPF5-SS. 
Table 30. The Tabular representation of CPF4-SS of example 11.

\begin{tabular}{|c|c|c|c|}
\hline$(\boldsymbol{\Psi},(\boldsymbol{\Delta}, \mathfrak{R}, \mathbf{4}))$ & $\mathfrak{r}_{\mathbf{1}}$ & $\mathfrak{r}_{2}$ & $\mathfrak{r}_{3}$ \\
\hline $\mathbf{3}_{1}$ & $2,\left(\begin{array}{c}0.75 e^{i 2 \pi(0.7)}, \\
0.09 e^{i 2 \pi(0.2)}, \\
0.15 e^{i 2 \pi(0.05)}\end{array}\right)$ & $3,\left(\begin{array}{c}0.8 e^{i 2 \pi(0.85)}, \\
0.09 e^{i 2 \pi(0.05)}, \\
0.1 e^{i 2 \pi(0.09)}\end{array}\right)$ & $3,\left(\begin{array}{c}0.85 e^{i 2 \pi(0.8)}, \\
0.1 e^{i 2 \pi(0.1)}, \\
0.03 e^{i 2 \pi(0.09)}\end{array}\right)$ \\
\hline $3_{2}$ & $1,\left(\begin{array}{c}0.25 e^{i 2 \pi(0.3)}, \\
0.07 e^{i 2 \pi(0.1)}, \\
0.25 e^{i 2 \pi(0.15)}\end{array}\right)$ & $0,\left(\begin{array}{c}0.15 e^{i 2 \pi(0.2)}, \\
0.06 e^{i 2 \pi(0.08)}, \\
0.35 e^{i 2 \pi(0.3)}\end{array}\right)$ & $3,\left(\begin{array}{c}0.86 e^{i 2 \pi(0.75)} \\
0.09 e^{i 2 \pi(0.1)} \\
0.03 e^{i 2 \pi(0.09)}\end{array}\right)$ \\
\hline $3_{3}$ & $3,\left(\begin{array}{c}0.9 e^{i 2 \pi(0.8)}, \\
0.04 e^{i 2 \pi(0.08)} \\
0.05 e^{i 2 \pi(0.1)}\end{array}\right)$ & $2,\left(\begin{array}{c}0.6 e^{i 2 \pi(0.55)}, \\
0.05 e^{i 2 \pi(0.05)}, \\
0.2 e^{i 2 \pi(0.09)}\end{array}\right)$ & $3,\left(\begin{array}{c}0.8 e^{i 2 \pi(0.78)} \\
0.03 e^{i 2 \pi(0.1)}, \\
0.1 e^{i 2 \pi(0.03)}\end{array}\right)$ \\
\hline
\end{tabular}

The tabular representation of CPP is demonstrated in table 31. We make the comparison table for CPP which is demonstrated in table 32. Afterwards, we determine the CPP score for each football team the with sum of grades $\left(\sum_{i=1}^{3} \mathfrak{x}_{\mathfrak{r}_{i}}\right)$ by subtracting the column sum from the row sum of table 32 and demonstrated in table 33.

Table 31. The tabular representation of CPP of example 11.

\begin{tabular}{|c|c|c|c|}
\hline $\boldsymbol{\phi}$ & $\mathfrak{r}_{\mathbf{1}}$ & $\mathfrak{r}_{\mathbf{2}}$ & $\mathfrak{r}_{\mathbf{3}}$ \\
\hline $\mathbf{z}_{1}$ & $0.75 e^{i 2 \pi(0.7)}$ & $0.8 e^{i 2 \pi(0.85)}$ & $0.85 e^{i 2 \pi(0.8)}$ \\
\hline $\mathbf{z}_{2}$ & $0.25 e^{i 2 \pi(0.3)}$ & $0.15 e^{i 2 \pi(0.2)}$ & $0.86 e^{i 2 \pi(0.75)}$ \\
\hline $\mathbf{z}_{3}$ & $0.9 e^{i 2 \pi(0.8)}$ & $0.6 e^{i 2 \pi(0.55)}$ & $0.8 e^{i 2 \pi(0.78)}$ \\
\hline
\end{tabular}

Table 32. The comparison table of CPP of example 11.

\begin{tabular}{|c|c|c|c|}
\hline & $3_{1}$ & 32 & 33 \\
\hline $\mathbf{z}_{1}$ & 3 & 2 & 2 \\
\hline $\mathbf{3}_{2}$ & 1 & 3 & 1 \\
\hline $\mathbf{3}_{3}$ & 1 & 2 & 3 \\
\hline
\end{tabular}

Table 33. The CPP score of each team.

\begin{tabular}{|c|c|c|c|c|}
\hline & $\begin{array}{c}\text { Grade sum } \\
\left(\sum_{i=1}^{\mathbf{3}} \mathfrak{x}_{\mathfrak{r}_{i}}\right)\end{array}$ & $\begin{array}{c}\text { Row sum } \\
\left(\mathcal{R} \boldsymbol{S}_{\mathbf{1}}\right)\end{array}$ & $\begin{array}{c}\text { Column sum } \\
\left(\mathcal{C} \boldsymbol{S}_{\mathbf{1}}\right)\end{array}$ & $\nabla_{\mathbf{1}}=\mathcal{R S}_{\mathbf{1}}-\mathcal{C} \boldsymbol{S}_{\mathbf{1}}$ \\
\hline $\mathbf{z}_{\mathbf{1}}$ & 8 & 7 & 5 & 2 \\
\hline $\mathbf{3}_{2}$ & 4 & 5 & 7 & -2 \\
\hline $\mathbf{3}_{3}$ & 8 & 6 & 6 & 0 \\
\hline
\end{tabular}

Now similarly the tabular representation of $\mathrm{CnP}$ is demonstrated in table 34 . We make the comparison table for $\mathrm{CnP}$ which is demonstrated in table 35. Afterwards, we determine the $\mathrm{CnP}$ score for each football team with the sum of grades $\left(\sum_{i=1}^{3} \mathfrak{x}_{\mathfrak{r}_{i}}\right)$ by subtracting the column sum from the row sum of table 35 and demonstrated in table 36. 
Table 34. The tabular representation of CnP of example 11.

\begin{tabular}{|c|c|c|c|}
\hline $\boldsymbol{\varphi}$ & $\mathfrak{r}_{\mathbf{1}}$ & $\mathfrak{r}_{\mathbf{2}}$ & $\mathfrak{r}_{\mathbf{3}}$ \\
\hline $\mathbf{z}_{\mathbf{1}}$ & $0.09 e^{i 2 \pi(0.2)}$ & $0.09 e^{i 2 \pi(0.05)}$ & $0.1 e^{i 2 \pi(0.1)}$ \\
\hline $\mathbf{3} \mathbf{2}$ & $0.07 e^{i 2 \pi(0.1)}$ & $0.06 e^{i 2 \pi(0.08)}$ & $0.09 e^{i 2 \pi(0.1)}$ \\
\hline $\mathbf{3} 3$ & $0.04 e^{i 2 \pi(0.08)}$ & $0.05 e^{i 2 \pi(0.05)}$ & $0.03 e^{i 2 \pi(0.1)}$ \\
\hline
\end{tabular}

Table 35. The comparison table of CnP of example 11.

\begin{tabular}{|c|c|c|c|}
\hline & 31 & 32 & 33 \\
\hline $\mathbf{z}_{1}$ & 3 & 3 & 3 \\
\hline $\mathbf{3 2}_{2}$ & 0 & 3 & 3 \\
\hline $\mathbf{3}_{3}$ & 0 & 0 & 3 \\
\hline
\end{tabular}

Table 36. The CnP score of each team.

\begin{tabular}{|c|c|c|c|c|}
\hline & $\begin{array}{c}\text { Grade sum } \\
\left(\sum_{i=1}^{\mathbf{3}} \mathfrak{x}_{\mathbf{r}_{i}}\right)\end{array}$ & $\begin{array}{c}\text { Row sum } \\
\left(\mathcal{R} \boldsymbol{S}_{\mathbf{2}}\right)\end{array}$ & $\begin{array}{c}\text { Column sum } \\
\left(\mathcal{C} \boldsymbol{S}_{\mathbf{2}}\right)\end{array}$ & $\nabla_{\mathbf{2}}=\mathcal{R S}_{\mathbf{2}}-\boldsymbol{C S}_{\mathbf{2}}$ \\
\hline $\mathbf{3}_{\mathbf{1}}$ & 8 & 9 & 3 & 6 \\
\hline $\mathbf{3}_{2}$ & 4 & 6 & 6 & 0 \\
\hline $\mathbf{z}_{3}$ & 8 & 3 & 9 & -6 \\
\hline
\end{tabular}

Next the tabular representation of CNP is demonstrated in table 37. We make the comparison table for CNP which is demonstrated in table 38. Afterwards, we determine the CNP score for each football team with the sum of grades $\left(\sum_{i=1}^{3} \mathfrak{x}_{\mathfrak{r}_{i}}\right)$ by subtracting the column sum from the row sum of table 38 . and demonstrated in table 39.

Table 37. The tabular representation of CNP of example 11.

\begin{tabular}{|c|c|c|c|}
\hline $\boldsymbol{\psi}$ & $\mathfrak{r}_{\mathbf{1}}$ & $\mathfrak{r}_{\mathbf{2}}$ & $\mathfrak{r}_{\mathbf{3}}$ \\
\hline $\mathbf{z}_{\mathbf{1}}$ & $0.15 e^{i 2 \pi(0.05)}$ & $0.1 e^{i 2 \pi(0.09)}$ & $0.03 e^{i 2 \pi(0.09)}$ \\
\hline $\mathbf{z}_{2}$ & $0.25 e^{i 2 \pi(0.15)}$ & $0.35 e^{i 2 \pi(0.3)}$ & $0.03 e^{i 2 \pi(0.09)}$ \\
\hline $\mathbf{z}_{3}$ & $0.05 e^{i 2 \pi(0.1)}$ & $0.2 e^{i 2 \pi(0.09)}$ & $0.1 e^{i 2 \pi(0.03)}$ \\
\hline
\end{tabular}

Table 38. The comparison table of CNP of example 11.

\begin{tabular}{|c|c|c|c|}
\hline & $3_{1}$ & 32 & 33 \\
\hline $\mathbf{3 1}_{1}$ & 3 & 1 & 1 \\
\hline $\mathbf{3 2}_{\mathbf{3}}$ & 3 & 3 & 2 \\
\hline
\end{tabular}

Table 39. The CNP score of each team.

\begin{tabular}{|l|c|c|c|c|}
\hline & $\begin{array}{c}\text { Grade sum } \\
\left(\sum_{i=1}^{3} \mathfrak{x}_{\mathfrak{r}_{i}}\right)\end{array}$ & $\begin{array}{c}\text { Row sum } \\
\left(\mathcal{R} S_{3}\right)\end{array}$ & $\begin{array}{c}\text { Column sum } \\
\left(\mathcal{C} S_{3}\right)\end{array}$ & $\nabla_{3}=\mathcal{R S}_{3}-\mathcal{C} S_{3}$ \\
\hline
\end{tabular}




\begin{tabular}{|c|c|c|c|c|}
\hline $\mathbf{3} 1$ & 8 & 5 & 8 & -3 \\
\hline $\mathbf{3} 2$ & 4 & 8 & 5 & 3 \\
\hline $\mathbf{3}_{3}$ & 8 & 6 & 6 & 0 \\
\hline
\end{tabular}

At the end, add the scores of $\mathrm{CnP}$ and $\mathrm{CNP}$ which is displayed in table 40 and then subtract it by the score of PP as demonstrated in table 41.

Table 40. Addition of CnP and CNP scores of example 11.

\begin{tabular}{|c|c|c|c|c|}
\hline & $\begin{array}{c}\text { Grade sum } \\
\left(\sum_{i=1}^{3} \mathfrak{x}_{\mathfrak{r}_{i}}\right)\end{array}$ & $\boldsymbol{\nabla}_{\mathbf{2}}$ & $\boldsymbol{\nabla}_{\mathbf{3}}$ & $\boldsymbol{\nabla}_{\mathbf{4}}=\boldsymbol{\nabla}_{\mathbf{2}}+\boldsymbol{\nabla}_{\mathbf{3}}$ \\
\hline $\mathbf{z}_{1}$ & 8 & 6 & -3 & 3 \\
\hline $\mathbf{3}_{2}$ & 4 & 0 & 3 & 3 \\
\hline $\mathbf{3}_{3}$ & 8 & -6 & 0 & -6 \\
\hline
\end{tabular}

Table 41. Table of final score with grades of example 11.

\begin{tabular}{|c|c|c|c|c|}
\hline & $\begin{array}{c}\text { Grade sum } \\
\left(\sum_{i=1}^{\mathbf{3}} \mathfrak{x}_{\mathbf{r}_{i}}\right)\end{array}$ & $\boldsymbol{\nabla}_{\mathbf{1}}$ & $\boldsymbol{\nabla}_{\mathbf{4}}$ & $\begin{array}{c}\text { Final Score } \\
=\boldsymbol{\nabla}_{\mathbf{1}}-\boldsymbol{\nabla}_{\mathbf{4}}\end{array}$ \\
\hline $\boldsymbol{z}_{\mathbf{1}}$ & 8 & 2 & 3 & -1 \\
\hline $\mathbf{3}_{2}$ & 4 & -2 & 3 & -5 \\
\hline $\mathbf{3}_{3}$ & 8 & 0 & -6 & 6 \\
\hline
\end{tabular}

From table 41 we noted that the football team $z_{1}$ and $z_{3}$ has maximum grades but $z_{3}$ has the highest score than the team $z_{1}$ so from the audience poll we got that the team Brazil will win the world cup 2022.

\section{Comparison}

In this part of the article, we will do the comparison of our established work with existing work such as IFN-SS. Using the following example we will show that our established work CPFN-SS is more general and effective than the IFN-SS.

Example 12: Reconsider example 11 in section 4 where we conducted an audience poll to get the point of view of people about the winner of the world cup 2022. We used the data in the form of CPFN-SS and got the result that the team brazil will win the world cup of 2022. In example 11 some people cast their votes in favor of teams (which is the positive grade of these teams), some remain neutral i.e. maybe in the favor or against these teams (which is neutral grade), and some cast their votes against the teams (which is negative grade for these teams). If we neglect the neutral grade (the people who remain neutral) in the data given in example 11 then the data will transform in the form of complex intuitionistic fuzzy N-SS. Now if we take phase term zero in both positive and negative grades and neglect the neutral grade then the data will convert in the environment of intuitionistic fuzzy N-SS (IFN-SS) which is demonstrated in table 11.

Table 42. The tabular form of IF4-SS.

$(\boldsymbol{\Omega}, \mathfrak{R}, \mathbf{4})$

$\mathfrak{r}_{1}$

$\mathfrak{r}_{1}$

$\mathfrak{r}_{2}$

$\mathfrak{r}_{3}$ 


\begin{tabular}{|l|c|c|c|}
\hline $\mathbf{z}_{1}$ & $2,(0.75,0.15)$ & $3,(0.8,0.1)$ & $3,(0.85,0.03)$ \\
\hline $\mathbf{z}_{2}$ & $1,(0.25,0.25)$ & $0,(0.15,0.35)$ & $3,(0.86,0.03)$ \\
\hline $\mathbf{z}_{3}$ & $3,(0.9,0.05)$ & $2,(0.6,0.2)$ & $3,(0.8,0.1)$ \\
\hline
\end{tabular}

Now we will utilize the algorithm defined by Akram et al. [37] for solving data in the environment of IFNSS.

The tabular representation of positive pole (PP) is demonstrated in table 43. We make the comparison table for PP which is demonstrated in table 44. Afterwards, we determine the PP score for each football team with the sum of grades $\left(\sum_{i=1}^{3} \mathfrak{x}_{\mathbf{r}_{i}}\right)$ by subtracting the column sum from the row sum of table 44 and demonstrated in table 45 .

Table 43. The tabular representation of PP of example 12.

\begin{tabular}{|c|c|c|c|}
\hline $\boldsymbol{\phi}$ & $\mathfrak{r}_{\mathbf{1}}$ & $\mathfrak{r}_{2}$ & $\mathfrak{r}_{3}$ \\
\hline $\mathbf{3}_{1}$ & 0.75 & 0.8 & 0.85 \\
\hline $\mathbf{3}_{2}$ & 0.25 & 0.15 & 0.86 \\
\hline $\mathbf{3}_{3}$ & 0.9 & 0.6 & 0.8 \\
\hline
\end{tabular}

44. The comparison table of PP of example 12.

\begin{tabular}{|c|c|c|c|}
\hline. & $3_{1}$ & $3_{2}$ & $3_{3}$ \\
\hline $\boldsymbol{3}_{1}$ & 3 & 2 & 2 \\
\hline $\mathbf{z}_{2}$ & 1 & 3 & 1 \\
\hline $\mathbf{3}_{3}$ & 1 & 2 & 3 \\
\hline
\end{tabular}

Table 45. The PP sore of each team.

\begin{tabular}{|c|c|c|c|c|}
\hline & $\begin{array}{c}\text { Grade sum } \\
\left(\sum_{i=1}^{\mathbf{3}} \mathfrak{x}_{\mathfrak{r}_{i}}\right)\end{array}$ & $\begin{array}{c}\text { Row sum } \\
\left(\mathcal{R} \boldsymbol{S}_{\mathbf{1}}\right)\end{array}$ & $\begin{array}{c}\text { Column sum } \\
\left(\boldsymbol{C} \boldsymbol{S}_{\mathbf{1}}\right)\end{array}$ & $\boldsymbol{\nabla}_{\mathbf{1}}=\boldsymbol{R S}_{\mathbf{1}}-\boldsymbol{C} \boldsymbol{S}_{\mathbf{1}}$ \\
\hline $\boldsymbol{z}_{\mathbf{1}}$ & 8 & 7 & 5 & 2 \\
\hline $\boldsymbol{3}_{2}$ & 4 & 5 & 7 & -2 \\
\hline $\boldsymbol{3}_{3}$ & 8 & 6 & 6 & 0 \\
\hline
\end{tabular}

Next the tabular representation of negative pole (NP) is demonstrated in table 46 . We make the comparison table for NP which is demonstrated in table 47. Afterwards, we determine the NP score for each football team with the sum of grades $\left(\sum_{i=1}^{3} \mathfrak{x}_{\mathfrak{r}_{i}}\right)$ by subtracting the column sum from the row sum of table 47 and demonstrated in table 48.

Table 46. The tabular representation of NP of example 12.

\begin{tabular}{|c|c|c|c|}
\hline $\boldsymbol{\psi}$ & $\mathfrak{r}_{1}$ & $\mathfrak{r}_{2}$ & $\mathfrak{r}_{3}$ \\
\hline $\boldsymbol{3}_{1}$ & 0.15 & 0.1 & 0.03 \\
\hline $\mathbf{3}_{2}$ & 0.25 & 0.35 & 0.03 \\
\hline $\boldsymbol{3}_{3}$ & 0.05 & 0.2 & 0.1 \\
\hline
\end{tabular}


Table 47. The comparison table of NP of example 12.

\begin{tabular}{|c|c|c|c|}
\hline & $3_{1}$ & $3_{2}$ & $3_{3}$ \\
\hline $\mathbf{z}_{1}$ & 3 & 1 & 1 \\
\hline $\mathbf{3 2}_{2}$ & 3 & 3 & 2 \\
\hline $\mathbf{3}$ & 2 & 1 & 3 \\
\hline
\end{tabular}

Table 48. The NP score of each team.

\begin{tabular}{|c|c|c|c|c|}
\hline & $\begin{array}{c}\text { Grade sum } \\
\left(\sum_{i=1}^{\mathbf{3}} \mathfrak{x}_{\mathfrak{r}_{i}}\right)\end{array}$ & $\begin{array}{c}\text { Row sum } \\
\left(\mathcal{R} \boldsymbol{S}_{\mathbf{3}}\right)\end{array}$ & $\begin{array}{c}\text { Column sum } \\
\left(\mathcal{C} \boldsymbol{S}_{\mathbf{3}}\right)\end{array}$ & $\boldsymbol{\nabla}_{\mathbf{3}}=\mathcal{R S}_{\mathbf{3}}-\boldsymbol{C S}_{\mathbf{3}}$ \\
\hline $\mathbf{3}_{\mathbf{1}}$ & 8 & 5 & 8 & -3 \\
\hline $\mathbf{3}_{2}$ & 4 & 8 & 5 & 3 \\
\hline $\mathbf{3}_{3}$ & 8 & 6 & 6 & 0 \\
\hline
\end{tabular}

In the end, subtract the score of NP from score of PP which is demonstrated in table $49 .$.

Table 49. Final score with grades of example 12.

\begin{tabular}{|c|c|c|c|c|}
\hline & $\begin{array}{c}\text { Grade sum } \\
\left(\sum_{i=1}^{3} \mathfrak{x}_{\mathfrak{r}_{i}}\right)\end{array}$ & $\boldsymbol{\nabla}_{\mathbf{1}}$ & $\boldsymbol{\nabla}_{\mathbf{3}}$ & $\begin{array}{c}\text { Final Score } \\
=\boldsymbol{\nabla}_{\mathbf{1}}-\boldsymbol{\nabla}_{\mathbf{3}}\end{array}$ \\
\hline $\mathbf{3}_{\mathbf{1}}$ & 8 & & & 5 \\
\hline $\mathbf{3}_{2}$ & 4 & 2 & -3 & -5 \\
\hline $\mathbf{3}_{3}$ & 8 & -2 & 0 & 0 \\
\hline
\end{tabular}

By using the algorithm defined by Akram et al. [37] observed from table 49 that the football team $z_{1}$ and $z_{3}$ has maximum grades but $z_{1}$ has the highest score than the team $z_{3}$ so from the audience poll we got that the team Belgium will win the world cup 2022.

We know that the voters may be divided into 4 groups i.e. vote for a team, abstain or neutral, vote against the team, and refusal of the voting. As we noted in example 11 and 12 that both are giving different results, in example 11 we consider the information in the environment of CPFN-SS so we get the result that the team Brazil will win the world cup 2022 and in example 12 we neglect the complex neutral grade and also take one-dimensional information by taking the phase term zero so we get the result that the team Belgium will win the world cup 2022. From example 12 we can observe that IFN-SS cannot provide us an accurate answer and cannot carry two-dimensional information, but our established model PFN-SS can carry twodimensional information and complex neutral grade along with grades and provide the accurate answer. when a decision-maker gives such type of data like yes, no, abstain, or neutral along with two-dimensional information in grading system then the existing notions such as FN-SS, IFN-SS, etc. are not capable to cope with it. To resolve such type of complication the idea of CPFN-SS is a perfect tool. So our established model CPFN-SS is the generalization of these models and is more beneficial. 
Our algorithm established in section 4 is more general than the algorithm established by Akram et al. [37]. Through our established algorithm one can resolve the data of the form of FN-SS, IFN-SS, complex FN-SS, and complex IFN-SS.

\section{Conclusion}

We established in this article, a new model CPFN-SSs which is the fusion of CPFSs along with N-SSs and explained it by an example. We also elaborated a novel definition of CPFS and gave its operation such as complement, union, and intersection. Furthermore, We established a complement of CPFN-SS in the article along with example. After that, we initiated two types of union, and intersection and explained them through examples. Moreover, we defined an algorithm to solve the data in the shape of CPFN-SSs, and then we represent two real-world applications (performance assessment of e-WRPs and predication about the champions of FIFA world cup 2022 through audience poll) of our established model in DM to show the edges and utility of the CPFN-SSs. In the performance assessment of e-WRPs, we noted that $z_{1}$ is the best e-WRP for a company and in predication about the champions of FIFA world cup 2022 through audience poll we noted that the team $z_{3}$ will win the world cup 2022. Finally, we presented a comparison of our established model with some existing methods which shows that our established model is more general than existing ones.

In the future, we want to talk about N-SSs to modified the ideas of bipolar fuzzy sets [38], complex hesitant fuzzy set [39] etc. [40, 41].

Conflict of interest: About the publication of this manuscript the authors declare that they have no conflict of interest.

Data availability: The data utilized in this manuscript are hypothetical and artificial, and one can use these data before prior permission by just citing this manuscript.

Author's contribution: All authors contributed equally in this article.

\section{References}

1. Zadeh, L. A. (1996). Fuzzy sets. In Fuzzy sets, fuzzy logic, and fuzzy systems: selected papers by Lotfi A Zadeh (pp. 394-432).

2. Gorzałczany, M. B. (1987). A method of inference in approximate reasoning based on intervalvalued fuzzy sets. Fuzzy sets and systems, 21(1), 1-17.

3. Pawlak, Z., Grzymala-Busse, J., Slowinski, R., \& Ziarko, W. (1995). Rough sets. Communications of the ACM, 38(11), 88-95.

4. Pawlak, Z. (2012). Rough sets: Theoretical aspects of reasoning about data (Vol. 9). Springer Science \& Business Media.

5. Pawlak, Z., \& Skowron, A. (2007). Rudiments of rough sets. Information sciences, 177(1), 3-27.

6. Pawlak, Z., \& Skowron, A. (2007). Rough sets: some extensions. Information sciences, 177(1), $28-$ 40.

7. Pawlak, Z., \& Skowron, A. (2007). Rough sets and Boolean reasoning. Information sciences, 177(1), 41-73.

8. Riaz, M., \& Hashmi, M. R. (2019). Linear Diophantine fuzzy set and its applications towards multiattribute decision-making problems. Journal of Intelligent \& Fuzzy Systems, 37(4), 5417-5439.

9. Atanassov, K. (2016). Intuitionistic fuzzy sets. International Journal Bioautomation, 20, 1. 
10. Cuong, B. C., \& Kreinovich, V. (2014). Picture fuzzy sets. Journal of Computer Science and Cybernetics, 30(4), 409-420.

11. Abdullah, S., Khan, S., Qiyas, M., \& Chinram, R. (2021). A Novel Approach Based on Sine Trigonometric Picture Fuzzy Aggregation Operators and Their Application in Decision Support System. Journal of Mathematics, 2021.

12. Ramot, D., Milo, R., Friedman, M., \& Kandel, A. (2002). Complex fuzzy sets. IEEE Transactions on Fuzzy Systems, 10(2), 171-186.

13. Ramot, D., Friedman, M., Langholz, G., \& Kandel, A. (2003). Complex fuzzy logic. IEEE Transactions on Fuzzy Systems, 11(4), 450-461.

14. Tamir, D. E., Jin, L., \& Kandel, A. (2011). A new interpretation of complex membership grade. International Journal of Intelligent Systems, 26(4), 285-312.

15. Imtiaz, A., Shuaib, U., Alolaiyan, H., Razaq, A., \& Gulistan, M. (2020). On Structural Properties ofComplex Fuzzy Sets and Their Applications. Complexity, 2020.

16. Akram, M., Bashir, A., \& Garg, H. (2020). Decision-making model under complex picture fuzzy Hamacher aggregation operators. Computational and Applied Mathematics, 39(3), 1-38.

17. Mahmood, T., Ur Rehman, U., Ali, Z., \& Mahmood, T. (2020). Hybrid vector similarity measures based on complex hesitant fuzzy sets and their applications to pattern recognition and medical diagnosis. Journal of Intelligent \& Fuzzy Systems, (Preprint), 1-22.

18. Mahmood, T., Ur Rehman, U., Ali, Z., \& Chinram, R. (2020). Jaccard and Dice Similarity Measures Based on Novel Complex Dual Hesitant Fuzzy Sets and Their Applications. Mathematical Problems in Engineering, 2020.

19. Molodtsov, D. (1999). Soft set theory - first results. Computers \& Mathematics with Applications, 37(4-5), 19-31.

20. Maji, P. K., Biswas, R., \& Roy, A. (2003). Soft set theory. Computers \& Mathematics with Applications, 45(4-5), 555-562.

21. Ali, M. I., Feng, F., Liu, X., Min, W. K., \& Shabir, M. (2009). On some new operations in soft set theory. Computers \& Mathematics with Applications, 57(9), 1547-1553.

22. Maji, P. K., Biswas, R. K., \& Roy, A. (2001). Fuzzy soft sets.

23. Feng, F., Khan, M., Leoreanu-Fotea, V., Anis, S., \& Ajaib, N. (2016). Fuzzy soft set approach to ideal theory of regular AG-groupoids. Analele Universitatii" Ovidius" Constanta-Seria Matematica, 24(1), 263-288.

24. Maji, P. K., Biswas, R., \& Roy, A. R. (2001). Intuitionistic fuzzy soft sets. Journal of fuzzy mathematics, 9(3), 677-692.

25. Maji, P. K. (2009, December). More on intuitionistic fuzzy soft sets. In International workshop on rough sets, fuzzy sets, data mining, and granular-soft computing (pp. 231-240). Springer, Berlin, Heidelberg.

26. Deschrijver, G., \& Kerre, E. E. (2003). On the relationship between some extensions of fuzzy set theory. Fuzzy sets and systems, 133(2), 227-235.

27. Yang, X., Lin, T. Y., Yang, J., Li, Y., \& Yu, D. (2009). Combination of interval-valued fuzzy set and soft set. Computers \& Mathematics with Applications, 58(3), 521-527.

28. Khan, M. J., Kumam, P., Ashraf, S., \& Kumam, W. (2019). Generalized Picture Fuzzy Soft Sets and Their Application in Decision Support Systems. Symmetry, 11(3), 415.

29. Khan, A., Izhar, M., \& Khalaf, M. M. (2019). Generalised multi-fuzzy bipolar soft sets and its application in decision making. Journal of Intelligent \& Fuzzy Systems, 37(2), 2713-2725.

30. Fatimah, F., Rosadi, D., Hakim, R. F., \& Alcantud, J. C. R. (2018). N-soft sets and their decision making algorithms. Soft Computing, 22(12), 3829-3842.

31. Alcantud, J. C. R., \& Laruelle, A. (2014). Dis\&approval voting: a characterization. Social Choice and Welfare, 43(1), 1-10. 
32. Ma, X., Liu, Q., \& Zhan, J. (2017). A survey of decision making methods based on certain hybrid soft set models. Artificial Intelligence Review, 47(4), 507-530.

33. Herawan, T., \& Deris, M. M. (2009, September). On multi-soft sets construction in information systems. In International conference on intelligent computing (pp. 101-110). Springer, Berlin, Heidelberg.

34. Chen, S., Liu, J., Wang, H., \& Augusto, J. C. (2013). Ordering based decision making-a survey. Information Fusion, 14(4), 521-531.

35. Ali, M. I., Mahmood, T., Rehman, M. M. U., \& Aslam, M. F. (2015). On lattice ordered soft sets. Applied soft computing, 36, 499-505.

36. Akram, M., Adeel, A., \& Alcantud, J. C. R. (2018). Fuzzy N-soft sets: A novel model with applications. Journal of Intelligent \& Fuzzy Systems, 35(4), 4757-4771.

37. Akram, M., Ali, G., \& Alcantud, J. C. R. (2019). New decision-making hybrid model: intuitionistic fuzzy N-soft rough sets. Soft Computing, 23(20), 9853-9868.

38. Mahmood, T. (2020). A Novel Approach towards Bipolar Soft Sets and Their Applications. Journal of Mathematics, 2020, Article ID 4690808.

39. Chinram, R., Mahmood, T., Ur Rehman, U., Ali, Z., \& Iampan, A. (2021). Some Novel Cosine Similarity Measures Based on Complex Hesitant Fuzzy Sets and Their Applications. Journal of Mathematics, 2021.

40. Mahmood, T., Rehman, U. U., \& Ali, Z. (2020). Exponential and non-Exponential Based Generalized Similarity Measures for Complex Hesitant Fuzzy Sets with Applications. Fuzzy Information and Engineering, 1-33.

41. Zhang, B., Mahmood, T., Ahmmad, J., Khan, Q., Ali, Z., \& Zeng, S. (2020). Cubic q-Rung Orthopair Fuzzy Heronian Mean Operators and Their Applications to Multi-Attribute Group Decision Making. Mathematics, 8(7), 1125. 
Figures

Level 1

E-Waste Recycling Program Performance Evaluation

Level 2

Criteria
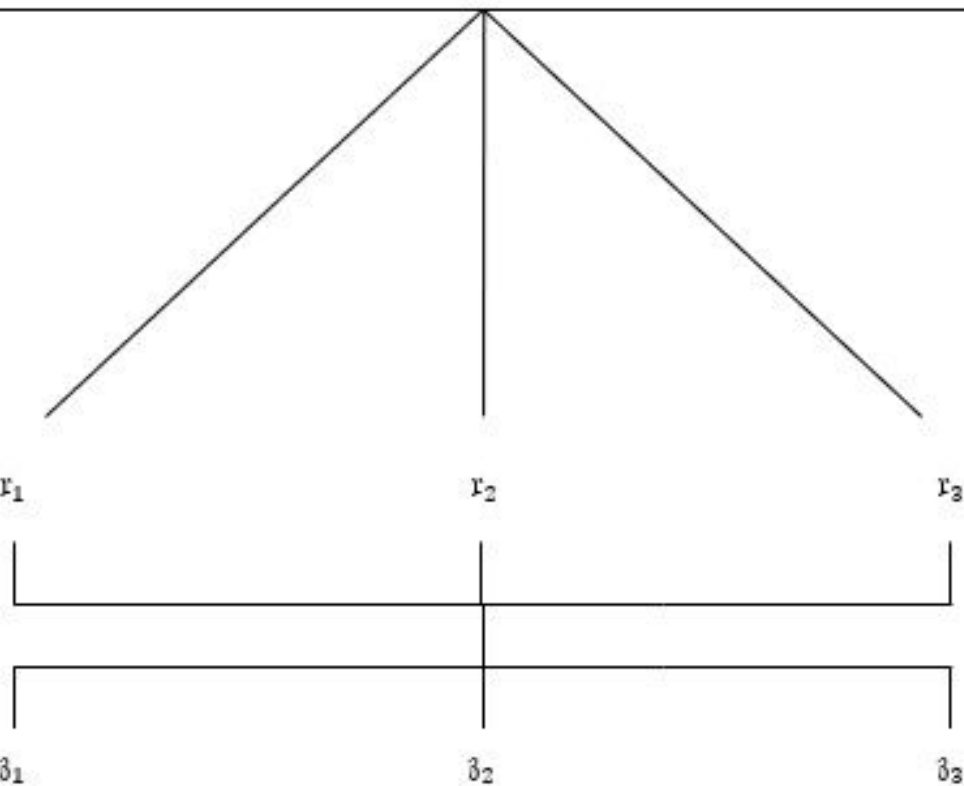

Legend

$\mathrm{r}_{1}$ : Social criteria $\quad \mathrm{x}_{2}=$ Technical criteria $\quad \mathrm{r}_{3}=$ Environmental criteria

$3_{i}(i=1,2, \ldots, n) \quad$ E-Waste Recycling Program

\section{Figure 1}

The hierarchical structure of e-WRP performance assessment. 